\title{
Fast 3-D Velocity Estimation in 4-D using a 62 + 62 Row-Column Addressed Array
}

Schou, Mikkel; Jørgensen, Lasse Thurmann; Beers, Christopher; Traberg, Marie Sand; Tomov, Borislav Gueorguiev; Stuart, Matthias Bo; Jensen, Jørgen Arendt

Published in:

IEEE Transactions on Ultrasonics, Ferroelectrics, and Frequency Control

Link to article, DOI:

10.1109/TUFFC.2020.3016991

Publication date:

2021

Document Version

Peer reviewed version

Link back to DTU Orbit

Citation (APA):

Schou, M., Jørgensen, L. T., Beers, C., Traberg, M. S., Tomov, B. G., Stuart, M. B., \& Jensen, J. A. (2021). Fast 3-D Velocity Estimation in 4-D using a $62+62$ Row-Column Addressed Array. IEEE Transactions on Ultrasonics, Ferroelectrics, and Frequency Control, 68(3), 608 - 623.

https://doi.org/10.1109/TUFFC.2020.3016991

\section{General rights}

Copyright and moral rights for the publications made accessible in the public portal are retained by the authors and/or other copyright owners and it is a condition of accessing publications that users recognise and abide by the legal requirements associated with these rights.

- Users may download and print one copy of any publication from the public portal for the purpose of private study or research.

- You may not further distribute the material or use it for any profit-making activity or commercial gain

- You may freely distribute the URL identifying the publication in the public portal 


\title{
Fast 3-D Velocity Estimation in 4-D using a $62+62$ Row-Column Addressed Array
}

\author{
Mikkel Schou $^{1}$, Lasse Thurmann Jørgensen ${ }^{1}$, Christopher Beers ${ }^{2}$, \\ Marie Sand Traberg ${ }^{1}$, Borislav Gueorguiev Tomov ${ }^{1}$, Matthias Bo Stuart ${ }^{1}$, and Jørgen Arendt Jensen ${ }^{1}$. \\ ${ }^{1}$ Center for Fast Ultrasound Imaging, Department of Health Technology, \\ Technical University of Denmark, DK-2800 Lyngby, Denmark \\ 2 BK Medical, 401 Science Park Road, State College, PA 16803, USA
}

\begin{abstract}
This paper presents an imaging scheme capable of estimating the full 3-D velocity vector field in a volume using rowcolumn addressed (RCA) arrays at a high volume rate. A $62+62$ RCA array is employed with an interleaved synthetic aperture sequence. It contains repeated emissions with rows and columns interleaved with B-mode emissions. The sequence contains 80 emissions in total and can provide continuous volumetric data at a volume rate above $125 \mathrm{~Hz}$. A transverse oscillation crosscorrelation estimator determines all three velocity components. The approach is investigated using Field II simulations and measurements using a specially built $3 \mathrm{MHz} 62+62 \mathrm{RCA}$ array connected to the SARUS experimental scanner. Both the B-mode and flow sequences have a penetration depth of $14 \mathrm{~cm}$ when measured on a tissue mimicking phantom $(0.5 \mathrm{~dB} /[\mathrm{MHz} \cdot \mathrm{cm}]$ attenuation). Simulations of a parabolic flow in a $12 \mathrm{~mm}$ diameter vessel at a depth of $30 \mathrm{~mm}$, beam-to-flow angle of $90^{\circ}$, and $x y$ rotation of $45^{\circ}$ gave a standard deviation (SD) of $(3.3,3.4,0.4) \%$ and bias of $(-3.3,-3.9,-0.1) \%$, for $\left(v_{x}, v_{y}, v_{z}\right)$. Decreasing the beam-to-flow angle to $60^{\circ}$ gave a SD of $(8.9,9.1,0.8) \%$ and bias of $(-7.6,-9.5,-7.2) \%$, showing a slight increase. Measurements were carried out using a similar setup, and pulsing at $2 \mathrm{kHz}$ yielded comparable results at $90^{\circ}$ with a SD of $(5.8,5.5,1.1) \%$ and bias of $(1.4,-6.4,2.4) \%$. At $60^{\circ}$ the SD was $(5.2,4.71 .2) \%$ and bias $(-4.6$, 6.9, -7.4)\%. Results from measurements across all tested settings showed a maximum SD of $6.8 \%$ and a maximum bias of $15.8 \%$, for a peak velocity of $10 \mathrm{~cm} / \mathrm{s}$. A tissue mimicking phantom with a straight vessel was used to introduce clutter, tissue motion, and a pulsating flow. The pulsating velocity magnitude was estimated across 10 pulse periods and yielded an SD of $10.9 \%$. The method was capable of estimating transverse flow components precisely, but underestimated the flow with small beam-to-flow angles. The sequence provided continuous data in both time and space throughout the volume, allowing for retrospective analysis of the flow. Moreover, B-mode planes can be selected retrospectively anywhere in the volume. This shows that tensor velocity imaging (full 3-D volumetric vector flow imaging) can be estimated in 4-D $(x, y, z, t)$ using only 62 channels in receive, making 4-D volumetric imaging implementable on current scanner hardware.
\end{abstract}

\section{INTRODUCTION}

Three dimensional ultrasound imaging has been investigated for many years [1]-[3]. Estimation in 4-D (space + time) of all three components of the blood velocity, henceforth referred to as tensor velocity imaging (TVI), can provide important clinical information, as showcased with MRI [4]. Tensor blood flow estimation using ultrasound has the potential to greatly improve monitoring of diseases, as the true flow patterns can be visualized and evaluated, instead of only the 2-D projection onto a plane. TVI has previously been achieved with 2-D fully addressed matrix arrays [5], [6]. Ideal focusing and beamforming is achieved when all elements can be accessed individually in transmit as well as in receive. However, this puts high demands on the hardware, as all elements have to be controlled by the scanner continuously. To accomplish this, either the use of large research scanners [7]-[9] or EGCgating [6] has been employed to account for the high data rates associated with these probes. The high demands on the hardware only increase, when flow has to be estimated, due to the high volume rate required. It is also difficult to maintain a low F-number in receive focusing using fully populated arrays, as their size is restricted by the number of elements.

Solutions such as 2-D sparse arrays and row-column arrays (RCAs) have been suggested to mitigate this problem. Sparse arrays have been investigated for many years [10], [11]. They maintain a low channel count by undersampling the 2-D array by connecting sparsely to fewer elements, [11][14]. Volumetric images can then be produced with greatly reduced channel count, although their reduced active surface area causes concerns regarding flow estimation. The loss of active area leads to a lower signal-to-noise ratio, with a loss up to 18 to $24 \mathrm{~dB}$ compared to the fully addressed 1024 element array for a 256 channel sparse array [15]. RCAs reduce the number of channel connections to the probe from $N^{2}$ to $2 N$ by addressing the rows and columns separately. $N$ is the number of elements along one side of the array [16]-[20]. RCAs can utilize the full surface area in transmit, as well as in receive, to maintain a high SNR. This is highly important in blood flow estimation, as scattering from blood is $40 \mathrm{~dB}$ lower than the surrounding tissue [21]. For a RCA, the number of channels scales linearly with the side-length, as opposed to fully addressed matrix probes. Theoretically, a RCA has an improved resolution compared to a fully connected 2-D array for the same number of channels, when the side-length is above $N>3$ [22] and the same element pitch is employed. The RCAs suffer slightly in terms of contrast [23], but offer a reduction in number of channels of $96 \%$ from $62^{2}=3844$ to $62 \cdot 2=124$ for a $62+62$ RCA probe. The use of advanced imaging techniques such as synthetic aperture imaging (SA), 
also known as coherent compounding imaging, can improve the image quality, especially in terms of contrast [24]-[26]. These have, successfully been employed on RCAs [18], [20], [22], [27].

Velocities in 2-D can be estimated with ultrasonic vector flow imaging (VFI), utilizing either: vector doppler, [28], echo particle image velocimetry [29], Blood speckle tracking [30] or transverse oscillation (TO) [31]. Here, TO can be used to estimate the transverse flow components by introducing a double oscillating field.

RCAs can be used in estimating vector flow in 3-D in either a plane [32], or expanding it to a volume [33] with steered emissions, both utilizing TO. This approach limits the volume rate, and B-mode images are difficult to achieve simultaneously. An improved scheme could employ SA flow imaging [26], [34]. SA B-mode imaging was presented for a flat RCA in [22], showing that an acceptable image quality could be attained using only 62 rows and 62 columns. Power Doppler estimation was also implemented on a large $128+128$ elements RCA with plane wave emissions [27], but grating lobes were here at a level of $-5 \mathrm{~dB}$ with 4 transmit events. The method was refined in [35] where it was used in functional brain imaging. Power Doppler is a qualitative measure of blood flow Power, and no directional information is readily obtained through the measure.

This paper investigates whether TVI (full volumetric vector flow) and anatomic imaging can be attained simultaneously, using a prototype $62+62$ RCA [36] in combination with SA imaging. An interleaved emission sequence is used in combination with the cross-correlation directional TO estimation approach for attaining a high maximum detectable velocity [37], [38]. The imaging scheme has been optimized for investigating the carotid artery with focusing optimized close to the probe and estimation of velocities up to $2 \mathrm{~m} / \mathrm{s}$ in the lateral direction.

The approach was previously presented in a conference paper [39], however the full performance is yet to be investigated. In this paper the approach is investigated both through Field II simulations [40], [41] and measurements using the experimental SARUS scanner [9] in combination with a prototype $62+62$ piezoelectric RCA [36] for stationary and pulsating flow.

\section{TheORY OF Row-COLUMN IMAGING}

RCA can conceptually be thought of as two orthogonal 1D arrays with overlapping regions allowing for 3-D imaging [20]. However, elements can only be accessed from either their rows or columns, and not individually. Designing sequences for RCAs therefore requires thorough understanding of the RCA processing scheme. This section presents some of the difficulties and solutions to achieve 3-D volumetric imaging using RCAs from previous work in terms of focusing, imaging, and velocity estimation.

\section{A. Focusing using RCAs}

The row and column addressing provide transmit steering either along rows or columns. Applying a delay curve along the transmitting elements generates a cylindrical wave that converges in a focus line rather than a focus point. With this assumption, and with the use of a specialized beamformer, 3-D imaging with good performance was possible [20].

Three dimensional images are achieved, when reception is made with the elements orthogonal to the transmitting elements. Transmitting and receiving with the same elements will not provide any 3-D information, as the received echoes will be an integration of echoes along the elongated elements. Transmission should therefore be performed with one side (rows or columns), and reception with the other side (the orthogonal elements) to achieve 3-D information. This results in only $N$ signals being passed from the probe to the beamforming hardware, as only one side is sampled per emission.

However, the sequence design is limited by this, as there is no dynamic focusing along the direction of transmit with a single transmit event [22]. Plane-by-plane imaging introduces a transmit focus and only beamforms the plane orthogonal to the transmitted focused wave. Volumetric images are then generated by a sliding aperture approach, limiting the framerate as the entire volume has to be covered by emissions. An alternative approach is to employ SA imaging, which provides full continuous volumetric data, which is ideal for flow estimation. This method is described in the next section.

\section{B. Synthetic Aperture Imaging using RCAs}

SA imaging provides continuous data throughout the image [26], and synthesizes the transmit focus to achieve images that are focused dynamically in both transmit and receive. SA imaging with RCA works similar to 2-D SA imaging, as a full low resolution volume (LRV) is beamformed for each transmit. Each transmit focus line is labeled a virtual source line (VSL) and is a 3-D equivalent to the 2-D virtual source [26]. A VSL is defined by the line segment $\overline{A B}$ by its two endpoints $(A, B)$ :

$$
\operatorname{VSL}(i)=\left(\begin{array}{ll}
x_{A}(i) & x_{B}(i) \\
y_{A}(i) & y_{B}(i) \\
z_{A}(i) & z_{B}(i)
\end{array}\right)
$$

VSLs are parallel to either row elements or column elements. When rows are used as transmitters the VSL endpoints are defined as:

$$
\begin{aligned}
x_{A}(i) & =-x_{B}(i)=L / 2, \\
y_{A}(i) & =y_{B}(i)=y(i), \\
z_{A}(i) & =z_{B}(i)=z_{f},
\end{aligned} \quad \text { for } i \in\{1,2, \ldots M\} .
$$

with $z_{f}$ being the focal length, $L$ is the length of an element, i.e. the side-length of the array. $M$ is the total number of transmit events used for a single volume. When columns are used as transmitters the endpoints are defined as:

$$
\begin{aligned}
x_{A}(i) & =x_{B}(i)=x(i), \\
y_{A}(i) & =-y_{B}(i)=L / 2, \\
z_{A}(i) & =z_{B}(i)=z_{f} .
\end{aligned}
$$

Either the full aperture (either rows or columns) is used in transmit, in a phased array imaging setup, or a smaller subaperture can be employed for a sliding aperture approach instead. The F-number (F\#) is then the ratio of $z_{f}$ to the size of the active aperture. 

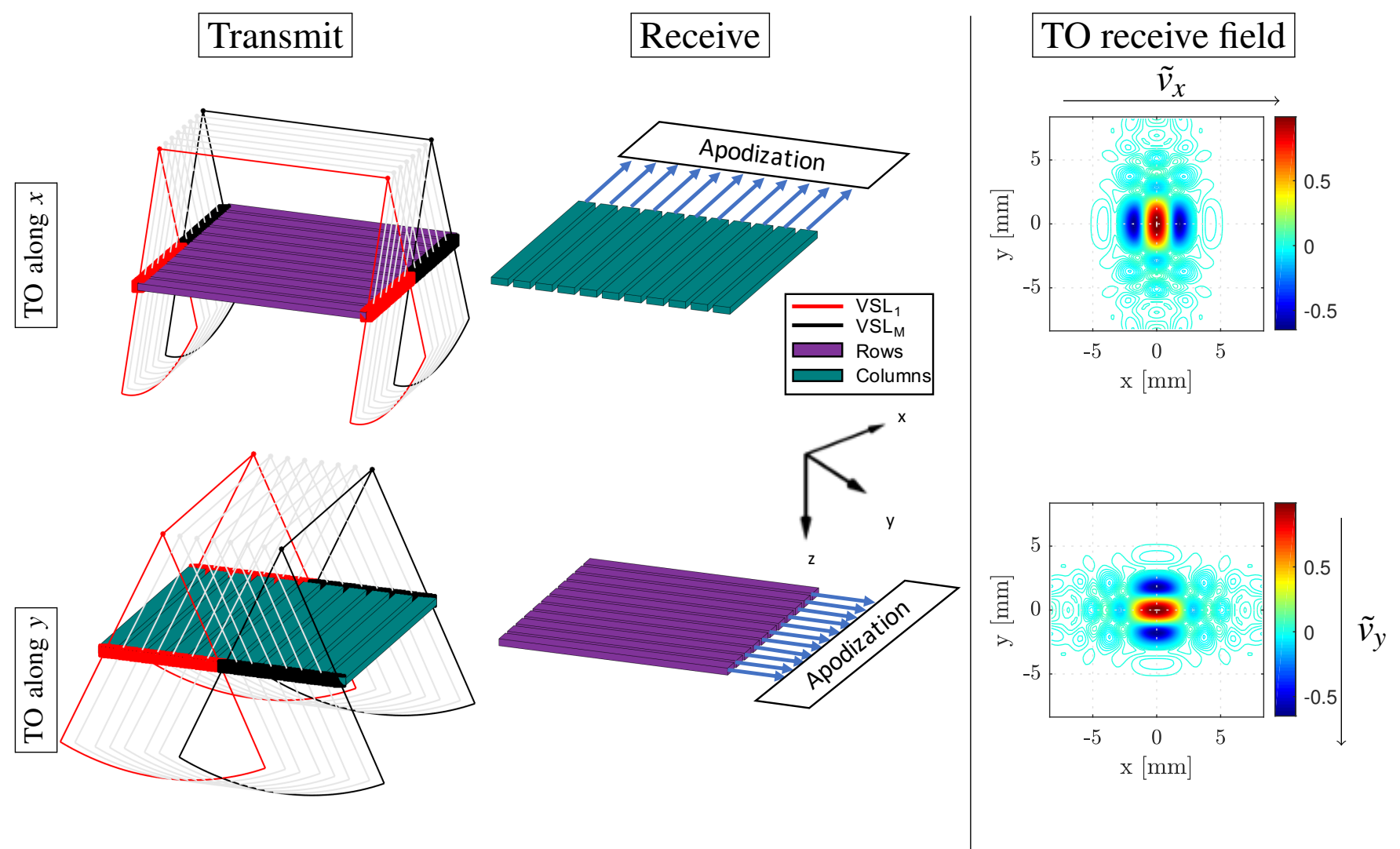

Fig. 1: Transmission and reception for eight VSL emissions along rows and columns. The two outermost emissions VSL $\mathrm{VS}_{1}$ and $\mathrm{VSL}_{M}$ are colored red and black, respectively; the rest are colored light gray. Applying a dual peak apodization along columns will provide a TO along $x$, used to estimate $v_{x}$. In the orthogonal set, the dual peak apodization along rows provides a TO in the $y$ direction, used to estimate $v_{y}$. The resulting receive field is visualized for a C-plane at $30 \mathrm{~mm}$, using a simulated setup. It shows the oscillating receive field (real part of the in-phase quadrature signal) for a stationary point on a linear scale, normalized to the maximum response. Please note, that each TO receive field is the result of the beamformed and summed contributions (LRVs), when the apodization in Fig. 2 is applied before beamformation. Here, $M=8$, as in the flow blocks.

The field is not focused by an elevation lens, and the entire volume along the emitting elements will be insonified as visualized in Fig. 1, resulting in a cylindrical wave. Here only the two outermost VSLs of the flow sequence $(i=1$ and $i=\mathrm{M})$ are shown. All VSLs are placed behind the transducer elements to ensure defocused waves with high overlap. The result of switching the transmit direction is also visualized. Each emission is then sampled using the orthogonal array and beamformed to reveal LRVs with different transmit waves. Each LRV is dynamically focused in receive but lacks transmit focus. Summing multiple LRVs results in a dynamically focused high resolution volume (HRV);

$$
\mathrm{HRV}=\sum_{i=1}^{M} W(x, y, z, i) \cdot \operatorname{LRV}(x, y, z, i),
$$

which is also synthetically focused in transmit. Here $W$ is an apodization matrix for side-lobe suppression. Note that $W$ is applied after beamformation. For B-mode a dynamic expanding apodization function can be applied to maintain a constant resolution in the entire field of view.

Optimizing for flow requires as few transmit events as possible for the highest possible volume rate. However, image quality is a compromise between number of transmit events and steering angles [22]. To attain the best possible resolution, the VSLs should be spread out over the full array, and a sufficient number of sources should be employed to attain low side lobes [42], [43].

An F-number of -1 for emitting defocused waves was chosen to ensure high spatial overlap, and simulations showed that 24 VSLs gave the best possible FWHM and lowest side lobes for the B-mode image. Eight VSLs per HRV were likewise selected as a compromise between frame rate and image contrast for data used in the flow estimation.

\section{Velocity eStimation USING RCAS}

Previously [32] demonstrated that RCAs can provide 3-D velocity estimates along a single M-mode. With electronic steering and multiple transmit events across the entire volume, even the full TVI estimate could be found [33]. The velocity was estimated using the TO method by switching between rows and columns in transmit, but this posed a problem as a high number of transmit events were required. SA with RCAs have been employed in an effort to lower the number of required transmit events, and achieve flow information [27], 


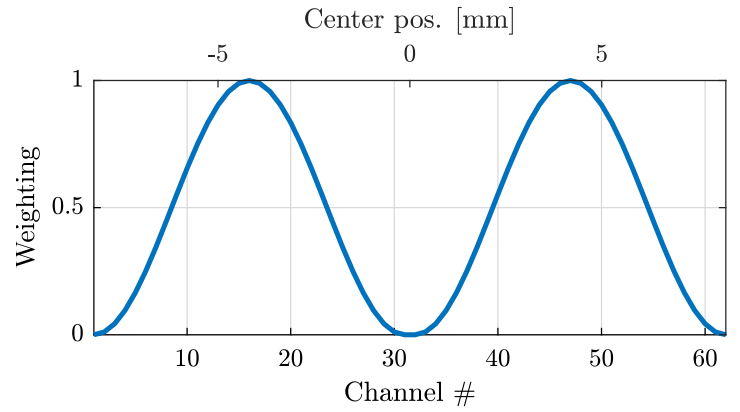

Fig. 2: Dual peak apodization used in receive to introduce a transverse oscillation receive field.

[35], [44]. However, only the power Doppler energy was estimated, which is inherently a one dimensional view of the flow. This study utilizes SA imaging and Directional Transverse Oscillations (DTO) [37], [38] to provide full volumetric information, and thereby estimate the full velocity vector.

\section{A. Directional Transverse Oscillation}

The DTO approach estimates the axial and transverse components in 2-D. Lines are beamformed transverse to the axial direction with a dual peak (DP) receive apodization to create a TO along the line. The DP apodization is visualized in Fig. 2. The theoretical lateral wavelength can be derived as:

$$
\lambda_{x}=2 \lambda_{z} \frac{z_{0}}{d_{x}},
$$

using the Fraunhofer approximation [31]. Here, $d_{x}$ is the distance between the two peaks, $\lambda_{z}$ is the emitted wavelength (axial), and $z_{0}$ is the imaging depth. The lateral wavelength is larger than the axial wavelength, as $d_{x}$ often is smaller than twice the imaging depth. The lateral wavelength can be changed by varying $d_{x}$ during receive processing, but limited by the physical width of the aperture. The estimator includes a pre-processing stage to decompose signals suitable for axial and lateral velocity estimation, and the two velocity components are then estimated independently using the crosscorrelation between two consecutive HRVs [37].

To estimate velocity in 3-D requires oscillating fields in the three orthogonal directions $(x, y, z)$. Steering for RCAs is limited to rows and columns, also in receive. Therefore the two transverse components $\left(v_{x}, v_{y}\right)$ have to be estimated separately [32]. In the axial direction $(z)$ the natural oscillation of the propagating wave can be used to estimate $v_{z}$, but each transverse component $v_{x}, v_{y}$ (transverse to the transducer surface) requires a transverse oscillation along $x$ and $y$, respectively. This is achieved by applying the DP given in Fig. 2 directly to the channel data before beamformation. Applying the DP apodization along the columns in receive, while transmitting with rows, provides an oscillating field along $x$ as visualized in Fig. 1. This oscillation is present in the final HRV as visualized by the beamformed and summed C-panel to the right. To estimate $v_{y}$ the oscillating field has to occur along $y$, and thereby requires the DP apodization to be applied to the rows, while transmitting with columns.
A total of four HRVs are required to estimate the full 3-D vector field: $\mathrm{HRV}_{a}^{R}$ and $\mathrm{HRV}_{b}^{R}$ are generated using rows in transmit, and $\mathrm{HRV}_{a}^{C}$ and $\mathrm{HRV}_{b}^{C}$ use columns. A total of $M$ transmissions are required to make each HRV. However, interleaving the emissions avoids a large temporal spacing between the HRVs to increase the maximum detectable velocity [37], and the following section describes this method.

\section{B. Interleaved SA emissions for flow}

The largest detectable velocity $\left(v_{\max }\right)$ possible to estimate is proportional to $\lambda f_{p r f, e f f}$, where $f_{p r f, e f f}$ is the effective pulse repetition frequency between high resolution volumes, and $\lambda$ is the wavelength. Two consecutive HRVs are usually made separately as:

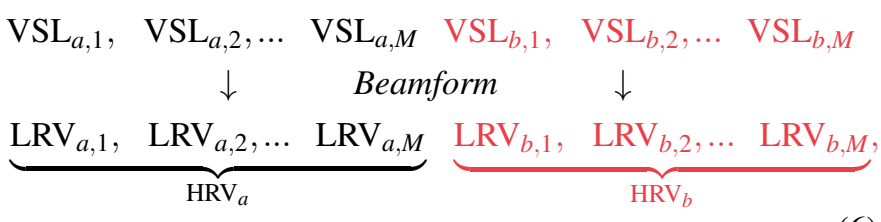

with the $b$ sequence being acquired exactly as $a$. Here $f_{p r f, e f f}$ is equal to $f_{p r f} / M$, where $f_{p r f}$ is the pulse repetition frequency. The maximum detectable velocity is thus reduced by a factor $M$ compared to the theoretical maximum. Interleaving the emissions causes a greater spatio-temporal overlap of moving targets in two consective HRVs [37], [38]:

$$
\underbrace{\mathrm{LRV}_{a, 1}, \overbrace{\mathrm{LRV}_{b, 1}, \quad \mathrm{LRV}_{a, 2}, \quad \mathrm{LRV}_{b, 2}, \ldots \quad \mathrm{LRV}_{a, M}}^{\mathrm{HRV}_{b}}, \mathrm{LRV}_{b, M}}_{\mathrm{HRV}_{a}},
$$

The time difference between $\mathrm{HRV}_{a}$ and $\mathrm{HRV}_{b}$ is reduced to $T_{p r f}=1 / f_{p r f}$, and the maximum detectable velocity is thereby increased to be closer to $v_{\max }=\lambda f_{p r f}$. One drawback of the approach is, however, that the HRVs are acquired over a longer duration, and this influences the coherence of the summed LRVs, which can affect the velocity estimation. This is investigated in Section V-B.

\section{Sequence design}

The sequence is designed for simultaneous flow and B-mode imaging with an optimized number of emissions. Interleaving and reusing emissions are an essential part of this optimization.

The sequence is comprised of two main types of blocks namely flow blocks and B-mode blocks, and is visualized in Fig. 3. The sequence includes two types of flow blocks; flow block $\mathrm{R}$ using rows for emissions and flow block $\mathrm{C}$ using columns. Each flow block spans $8+8=16$ emissions, which result in two HRVs per block. The sequence is emitted as:

$$
\begin{array}{lr}
\mathrm{LRV}_{a, 1}^{R}, \mathrm{LRV}_{b, 1}^{R}, \ldots, \mathrm{LRV}_{a, 8}^{R}, \mathrm{LRV}_{b, 8}^{R} & \text { (Flow block } R) \\
\mathrm{LRV}_{B, 1}^{R}, \mathrm{LRV}_{B, 2}^{R}, \mathrm{LRV}_{B, 3}^{R}, \mathrm{LRV}_{B, 4}^{R} & (B \text {-mode) } \\
\mathrm{LRV}_{a, 1}^{C}, \mathrm{LRV}_{b, 1}^{C}, \ldots, \mathrm{LRV}_{a, 8}^{C}, \mathrm{LRV}_{b, 8}^{C} & \text { (Flow block } C)
\end{array}
$$




$$
\begin{array}{cr}
\mathrm{LRV}_{B, 5}^{R}, \mathrm{LRV}_{B, 6}^{R}, \mathrm{LRV}_{B, 7}^{R}, \mathrm{LRV}_{B, 8}^{R} & (\text { (B-mode) } \\
\ldots & (\text { Flow block } R) \\
\mathrm{LRV}_{B, 9}^{R}, \mathrm{LRV}_{B, 10}^{R}, \mathrm{LRV}_{B, 11}^{R}, \mathrm{LRV}_{B, 12}^{R} & (\text { B-mode }) \\
\ldots & (\text { Flow block } C) \\
\mathrm{LRV}_{B, 13}^{R}, \mathrm{LRV}_{B, 14}^{R}, \mathrm{LRV}_{B, 15}^{R}, \mathrm{LRV}_{B, 16}^{R} & (B \text {-mode }) .
\end{array}
$$

The sequence produces a single volumetric B-mode, and two volumetric flow estimates as flow block $\mathrm{R}+\mathrm{C}$ combine to a single volumetric estimate. Each low resolution volume $\left(L R V_{a}^{*}\right)$ corresponds to a VSL transmission, reception, and beamforming, and $L R V_{b}^{*}$ is the repetition to fulfill (7). Superscript $R$ stands for rows transmission, and $C$ for columns transmission. Subscript $B$ are the designated B-mode emissions and are acquired with rows as transmitters.

Following (2), the virtual line positions are defined as:

$$
y(i)=(2 i-M-1) \cdot Q \cdot p_{s},
$$

where $Q$ is scalar, $p_{s}$ is the element pitch of the array, and $i$ denotes the index of the $M$ emissions. For the emissions in flow block $\mathrm{R}, M=8$ and $Q=2.2$. Initially, the B-mode emissions were also calculated using (8), with $\mathrm{M}=24$ and $Q=1$. However, the VSLs positions at $\mathrm{i}=[5,7,9,11,14$, $16,18,20]$ are not used. Instead, these emissions are replaced by emissions from flow block R, which have VSLs positions which lay reasonably close to the removed VSLs. The distance between VSLs remains low enough to ensure no grating lobes in the transmit direction for B-mode, while maintaining a low number of emissions. For flow block $\mathrm{C} y(i)$ is changed to $x(i)$ in (8). The effective virtual source pitch for flow and B-mode are listed in Table I together with other probe and imaging parameters.

The flow HRVs are denoted $\mathrm{HRV}_{a}^{R}$ (gray in Fig. 3) and $\mathrm{HRV}_{b}^{R}$ (green). For flow block $\mathrm{R}$, these are generated with 8 unique VSLs along rows and replicated once. The same VSLs are used for flow block $\mathrm{C}$, only rotated $90^{\circ}$, resulting in $\mathrm{HRV}_{a}^{C}$ (blue) and $\mathrm{HRV}_{b}^{C}$ (pink). The HRVs are then correlated using the method described in Section III-D.

The full sequence consists of four flow blocks with four Bmode emissions in between each block. This symmetry is enforced to avoid non-uniform temporal spacing. The sequence therefore spans $(16+4) \cdot 4=80$ emissions including the Bmode emissions. Pulsing at $10 \mathrm{kHz}$ then provides a B-mode volume rate of $125 \mathrm{~Hz}$, and a VFI volume rate of $250 \mathrm{~Hz}$. For one full volume estimate a flow block $\mathrm{R}$ and a flow block $\mathrm{C}$ is required. A single volumetric B-mode is acquired with the full 80 emissions.

\section{Implementation of the DTO velocity estimator}

Using flow block $\mathrm{R}, v_{x}$ was estimated for each point $\left(x_{p}, y_{p}, z_{p}\right)$ in the volume. This was performed by beamforming lines along the $x$-direction in $\operatorname{HRV}_{a}^{R}$ and $\mathrm{HRV}_{b}^{R}$ denoted $g_{a}(k)$ and $g_{b}(k)$. The sample distance was $\lambda_{x} / 8$, and a total of
$P=97$ points were beamformed. A pre-processing stage from [37], [38] removed the influence from the axial motion and yielded a set of new signals $g_{a}^{*}(k)$ and $g_{b}^{*}(k)$, which were crosscorrelated:

$$
R_{a b}\left(l_{x}, y_{p}, z_{p}\right)=\sum_{k=-(P-1) / 2}^{(P-1) / 2} g_{a}^{*}\left(k, y_{p}, z_{p}\right) g_{b}^{*}\left(k+l_{x}, y_{p}, z_{p}\right) .
$$

A total of $2 H+1$ by $2 H+1$ parallel lines were beamformed and correlated, and then averaged to minimize the influence of noise on the correlation function. $H$ was 4 , and a sampling density of $\Delta D=\lambda / 4$ was used in both $x$ and $y$. The average correlation function was then calculated as:

$$
R_{a v g, a b}\left(l_{x}\right)=\sum_{i_{y}=-H}^{H} \sum_{i_{z}=-H}^{H} R_{a b}\left(l_{x}, y_{p}+\Delta D \cdot i_{y}, z_{p}+\Delta D \cdot i_{z}\right) .
$$

Likewise, a set of correlations over time was also averaged. This will be referred to as correlation per estimate $C_{e s t}$. After this, the maximum interpolated lag, $l_{x, i n t}$, was found using a second-order polynomial fit to increase precision without oversampling the beamformed signals $g_{a}$ and $g_{b}$ [37], [45], which would otherwise significantly increase computation time.

The transverse velocity $\hat{v}_{x}$ along $x$ was then estimated as:

$$
\hat{v}_{x}=l_{x, i n t} \cdot T_{p r f} \cdot \Delta d
$$

where $\Delta d$ is the distance between two samples in meters along direction of the lines, here $x$, and $T_{p r f}$ is the time between pulse emissions. To estimate $v_{y}$ the transmit and receive directions are switched for flow block $\mathrm{C}$, having columns transmitting and DP apodization along the rows in receive. Similar processing applies to the y-direction by adapting equations (9)-(11) to match $v_{y}$ and $y$ instead.

\section{METHODS}

The imaging sequence was designed for a prototype $3 \mathrm{MHz}$, $\lambda / 2$-pitch, $62+62$ RCA piezoelectric probe with dimensions described in Table I [36]. The probe has integrated apodization, which is designed to prevent ghost artifacts of the main echo, by removing edge effects of the elongated elements [20]. Both simulations and measurements use the same sequence. Simulations use the measured impulse response, which is different between the row and the column arrays. Here follows a description of the simulation and measurement setups, data processing, and the method used in validating the performance of the estimator.

\section{A. Simulations - parameter study}

Stationary parabolic flow beneath the probe was simulated using Field II Pro [46]. A parabolic flow was simulated using a uniform distribution of scatterers placed in a volume. The parabolic flow was assumed to be laminar and modelled as:

$$
\vec{v}(r)=v_{\text {peak }}\left(1-\frac{r^{2}}{R^{2}}\right) .
$$



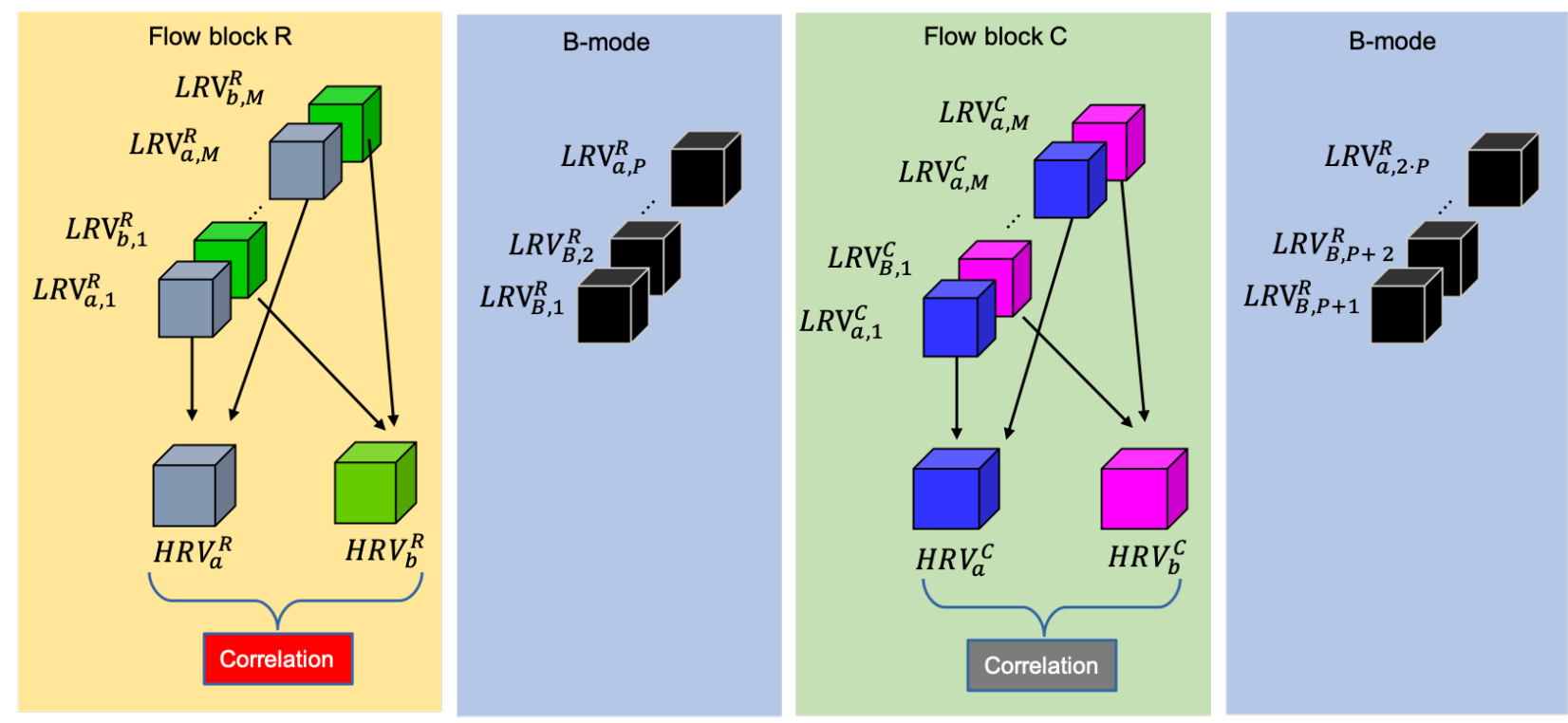

a)

(1)

(2)

Correlation

Fig. 3: a) shows the sequence graphically. Yellow block is flow block $\mathrm{R}$ used to acquire $\operatorname{HRV}_{a}^{R}$ and $\operatorname{HRV}_{b}^{R}$ by coherently summing all $\mathrm{LRV}_{a, i}^{R}$ and $\mathrm{LRV}_{b, i}^{R}$ using (4), respectively. Correlating the two HRVs provides a single 3-D correlation function (in red), which is used to estimate $v_{x}$ and $v_{z}$. Green block is flow block $\mathrm{C}$, where $\mathrm{HRV}_{a}^{C}$ and $\mathrm{HRV}_{b}^{C}$ are acquired by coherently summing all $\mathrm{LRV}_{a, i}^{C}$ and $\mathrm{LRV}_{b, i}^{C}$, respectively. $\mathrm{HRV}_{a, b}^{C}$ are then used to extract the correlation (in gray), which leads to estimate $v_{y}$ and $v_{z}$. The blue blocks are the designed B-mode transmissions. LRVs are combined respectively to form the specified HRV. b) shows how the correlations are temporally averaged to estimate the velocity.

TABLE I: Imaging settings and probe parameters

\begin{tabular}{l|c|l} 
Parameters & Name & Value \\
\hline \multicolumn{2}{c}{ Probe dimensions } \\
\hline Number of elements & $N$ & $62+62$ \\
Center frequency: & - & $3 \mathrm{MHz}$ \\
Kerf & - & $20 \mu \mathrm{m}$ \\
Pitch & $p_{r}$ & $270 \mu \mathrm{m}$ \\
Element length & - & $19 \mathrm{~mm}$ \\
Total surface area & - & $361 \mathrm{~mm}^{2}$ \\
Length of integrated apodization & - & $4.05 \mathrm{~mm}$ \\
\hline \multicolumn{2}{c}{ Imaging Parameters } \\
\hline Pulse repetition frequency & $f_{\text {prf }}$ & $2 \mathrm{kHz}$ \\
Transmit Apodization & - & von Hann $(\mathrm{F} \#=-1)$ \\
Synthetic Transmit apodization & $W(x, y, z)$ & von Hann $(\mathrm{F \#}=-1)$ \\
Dynamic Receive apodization & - & von Hann $(\mathrm{F} \#=1)$ \\
Flow apodization & - & Dual Peak \\
Receive sampling frequency & & $17.5 \mathrm{MHz}$ \\
Number of sequences & - & 400 \\
Number of active elements & - & 32 \\
No. transmitted cycles & - & 2 \\
Emitted center frequency & - & $3 \mathrm{MHz}$ \\
Focal length & $z_{f}$ & $-8.64 \mathrm{~mm}$ \\
Virtual source pitch B-mode & - & $540 \mu \mathrm{m}$ \\
Virtual source pitch Flow & - & $1188 \mu \mathrm{m}$ \\
Field of view, cross section & - & $17 \times 17 \mathrm{~mm}{ }^{2}$ \\
\hline
\end{tabular}

Here, $R$ is radius of the vessel, $r \in[0, R]$ is the radial coordinate of the vessel, and $v_{\text {peak }}$ peak velocity. From (12) the displace-
TABLE II: Parameters used in the simulation

\begin{tabular}{l|l|l}
\hline Parameter & name & Values \\
\hline \multicolumn{3}{c}{ Variable parameters } \\
\hline Beam-to-flow angle & $\alpha$ & $60^{\circ}, 75^{\circ}, 90^{\circ}$ \\
Flow angle, rotation in $x-y$ & $\beta$ & $0^{\circ}, 45^{\circ}$ \\
Pulse repetition frequency & $f_{\text {prf }}$ & $1,2,5, \mathbf{1 0} \mathrm{kHz}$ \\
Vessel center depth $(z)$ & - & $\mathbf{2 0}, 30 \mathrm{~mm}$ \\
Correlations per estimate & $C_{\text {est }}$ & $4,8, \mathbf{1 6}, 32,64$ \\
\hline \multicolumn{3}{c}{ Fixed parameters } \\
\hline Vessel radius & $R$ & $\mathbf{6} \mathrm{mm}$ \\
Speed of sound & - & $\mathbf{1 5 4 0} \mathrm{m} / \mathrm{s}$ \\
Vessel length (non-rotated) & - & $\mathbf{2 4} \mathrm{mm}$ \\
\hline
\end{tabular}

ment of the scatterers is easily calculated as: $\Delta \vec{d}(r)=T_{p r f} \vec{v}(r)$. The simulation parameter values are listed in Table II. The default parameters of the simulation are highlighted in bold. Assuming the non-rotated profile $\vec{v}(r)$ have $r=\sqrt{x^{2}+y^{2}}$, the rotated profile becomes:

$$
\vec{v}_{\text {true }}=\left[\begin{array}{c}
v(r) \sin (\alpha) \cos (\beta) \\
v(r) \sin (\alpha) \sin (\beta) \\
v(r) \cos (\alpha)
\end{array}\right],
$$

where $\alpha$ is the conventional beam-to-flow angle, and $\beta$ is the rotation of the vessel in the $x-y$ plane. 
A total of $160 \mathrm{HRVs}$ per parameter set were simulated to quantify the performance of the estimator. All simulations were evaluated against the true profiles in (12) and (13).

\section{B. Interleaved vs. non-interleaved}

Simulations with pure axial and pure lateral velocities were carried out to evaluate the effect of interleaving against non-interleaving. The non-interleaved sequence consisted of generating each HRV separately, and the interleaved sequence utilizes the emissions as in (7). The standard settings from Table II were used. Thus, the beam-to-flow angle was $90^{\circ}$ for lateral flow, and $0^{\circ}$ for the axial.

\section{Measurements}

All measurements were carried out using the $62+62$ RCA experimental probe connected to the experimental scanner SARUS [9]. The imaging parameters are shown in Table I for the sequence described in Section III-C. The measurements included 3 setups: A constant flow setup for validation, a tissue-mimicking phantom with pulsating flow, and lastly a speckle phantom for estimating penetration depth. Flow and B-mode images were processed separately as described in Section IV-E.

Flow-rig: Validation of the flow profiles were made with a custom made flow-rig system designed with a $1.2 \mathrm{~m}$ inlet to ensure a fully developed parabolic flow profile. A rubber vessel with a radius $(R)$ of $6 \mathrm{~mm}$ was submerged in water, and blood mimicking fluid was circulated inside the tube in a closed loop driven by a controllable pump. A magnetic flow meter (MAG 1100, Danfoss, Hasselager, Denmark) was attached to accurately estimate the volume flow rate $\mathrm{Q}$, which in turn can be translated to a peak velocity estimate by assuming parabolic flow [32]:

$$
v_{\text {peak }}=\frac{2 Q}{\pi R^{2}} .
$$

A custom made fixture allowed placement of the transducer relative to the rubber tube with the two rotations, $\alpha$ and $\beta$. A series of measurements were made with $\alpha=60^{\circ}, 75^{\circ}, 90^{\circ}$ and $\beta=0^{\circ}, 45^{\circ}$. The transducer was protected in a latex cover with gel to avoid water damage. It was then submerged in the water tank containing the rubber tube. The tank sides were covered with acoustical absorbing material to remove reverberation artefacts. The flow rate was set to attain a $v_{\text {peak }}$ of $10 \pm 0.01 \mathrm{~cm} / \mathrm{s}$ and $25 \pm 0.04 \mathrm{~cm} / \mathrm{s}$, by adjusting the flow pump settings to match the desired $Q$ using (14). A pulse repetition frequency of $2 \mathrm{kHz}$ was chosen to avoid reverberations in the flow rig.

Pulsatile flow: A phantom was modeled after an MRI scan of a healthy volunteer using polyvinyl alcohol (PVA) cryogel to mimic the properties of human tissue [47]. It contained a straight vessel structure from a common carotid artery scan [48]. The phantom was connected to a closedloop flow system, consisting of a programmable flow pump (CompuFlow 1000, Shelly Medicial Imaging Technologies, Toronto, Canada). Pulsating flow was generated with a carotid waveform mimicking the flow found in the artery. The peak volume flow rate was $10 \mathrm{~mL} / \mathrm{s}$, and the pulse period was $0.84 \mathrm{~s}$. The RCA transducer was positioned upstream of the bifurcation. Four hundred frames of the full sequence were recorded, providing 800 velocity estimates. $C_{e s t}$ was here set to 8 with a sliding window approach and $f_{p r f}$ of $2 \mathrm{kHz}$.

\section{Stationary Echo Removal}

An echo cancelling filter was implemented using subtraction of the mean signal as:

$$
H R V_{*, f l o w}(p)=H R V_{*}(p)-\frac{1}{2 K+1} \sum_{k=-K}^{K} H R V_{*}(p+k)
$$

Here, $\mathrm{HRV}_{*}$ denotes the high resolution volume, with $*$ being either $a$ or $b .2 K+1$ is the number of HRVs used in echocancelling. $K$ was set to 5 .

\section{E. Data Processing}

For each run of the sequence, channel data for $80 \mathrm{LRVs}$ were acquired, and the LRVs were beamformed offline using a specialized delay-and-sum beamformer [49]. The offline processing allowed for total flexibility of receive and virtual source apodization, and the same channel data could be beamformed multiple times with different apodization-schemes. The proposed sequence takes full advantage of this. When transverse flow is estimated, a fixed double peak apodization is applied in receive, and when axial flow is to be estimated or when performing B-mode imaging, dynamic apodization with an F-number of 1 is applied. The parameters for the different apodization schemes are summarized in Table I. Of the 80 emissions, 56 are used only for flow, 16 are used only for B-mode imaging, and 8 are used for both.

\section{F. Performance metrics}

The velocity estimator was evaluated based on the relative mean bias, $\tilde{B}$, and standard deviation, $\tilde{\sigma}$, of $N_{\text {est }}$ estimated velocity profiles of the axial line intersecting the center of the vessel at sample points $z$ inside the vessel. The mean profile $(\tilde{v})$ and standard deviation $(\sigma)$ was calculated over the $N_{\text {est }}$ velocity estimates, as [50]:

$$
\begin{aligned}
\tilde{v}\left(x_{0}, y_{0}, z\right) & =\frac{1}{N_{\text {est }}} \sum_{n=1}^{N_{\text {est }}} \hat{v}_{n}\left(x_{0}, y_{0}, z\right) \\
\sigma\left(x_{0}, y_{0}, z\right) & =\sqrt{\frac{1}{N_{\text {est }}-1} \cdot \sum_{n=1}^{N_{\text {est }}}\left(\hat{v}_{n}\left(x_{0}, y_{0}, z\right)-\tilde{v}\left(x_{0}, y_{0}, z\right)\right)^{2}}
\end{aligned}
$$

The relative measures $(\tilde{B})$ and $(\tilde{\sigma})$ were calculated as:

$$
\begin{aligned}
& \tilde{B}=\frac{1}{N_{\text {est }} \cdot v_{\text {peak }}} \sum_{z=1}^{N_{z}}\left(\tilde{v}\left(x_{0}, y_{0}, z\right)-v_{\text {true }}\left(x_{0}, y_{0}, z\right)\right) \\
& \tilde{\sigma}=\frac{1}{v_{\text {peak }}} \sqrt{\frac{1}{N_{z}} \sum_{j=1}^{N_{z}} \sigma\left(x_{0}, y_{0}, z\right)^{2}}
\end{aligned}
$$

Here $N_{z}$ are the discrete samples within the vessel along $z$, with $x_{0}=y_{0}=0$. Only the central $90 \%$ of the velocity profile 
was used in the calculations, due to potential echo-canceling filter effects at the border of the vessel. Profiles are visualized below with the mean using (16) colored red, $v_{\text {true }}$ blue, and the shaded gray area is $\bar{v}\left(x_{0}, y_{0}, z\right) \pm \sigma\left(x_{0}, y_{0}, z\right)$ to visually evaluate the performance. For the validation metrics where calculated for 10 independent estimated profiles $\left(N_{e s t}=10\right)$.

\section{RESUlts}

The full-width-half-maximum for the B-mode sequence was estimated using simulations, as: $(0.81,1.56,0.65) \mathrm{mm}=$ $(1.58 \lambda, 3.0 \lambda, 1.2 \lambda)$ for $(x, y, z)$ at a depth of $30 \mathrm{~mm}$, evaluated in two orthogonal planes going through the point. The penetration depth was estimated using a speckle phantom (Dansk Fantom Service, Denmark, model 571) with a $0.5 \mathrm{~dB} /[\mathrm{MHz}$ $\mathrm{cm}]$ attenuation. Twenty frames were acquired and used in the evaluation. The SNR was calculated for both the flow and Bmode sequences separately as a function of depth, and can be seen in Fig. 4. Lines were fitted using a linear model, showing an estimated penetration depth of $14 \mathrm{~cm}$ (B-fit and F-fit).

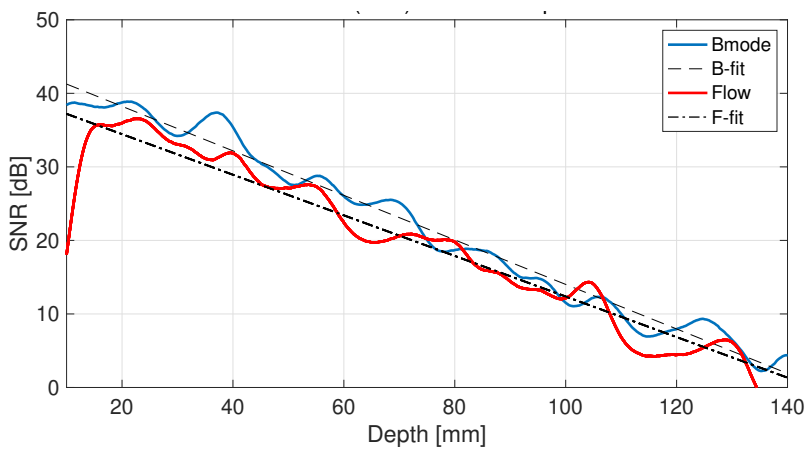

Fig. 4: Estimated SNR as a function of depth. Red shows the flow sequence and blue shows the B-mode sequence results. The SNR curves reach zero at approximately $140 \mathrm{~mm}$.

\section{A. Simulation results}

A subset of profiles from the full parameter study are shown in Fig. 5, where the three velocity components, $\left(v_{x}, v_{y}, v_{z}\right)$ are shown together with the magnitude $|v|=\sqrt{v_{x}^{2}+v_{y}^{2}+v_{z}^{2}}$. A rotation of $45^{\circ}$ in $x-y$ and varying beam-to-flow angles $(\alpha)$ are shown. All tested parameters from Table II are summarized in Fig. 6. Here $\tilde{B}$ and $\tilde{\sigma}$ of the three velocity components are shown against the varied parameter. The varied parameters are pulse repetition frequency $\left(f_{p r f}\right)$ as it relates to maximum detectable velocity, correlations per estimate $\left(C_{\text {est }}\right)$ as it relates to frame rate, and positioning of the probe in relation to the flow, i.e. depth and rotation $(\alpha, \beta)$.

Depth and rotation $(\alpha, \beta)$ : Fig. 6 (a) shows the bias and precision of the estimator with respect to every combination of $\alpha=(60,75,90)^{\circ}, \beta=(0,45)^{\circ}$ and depth $=(20,30) \mathrm{mm}$. The best results were found at $\alpha=90^{\circ}$; here the precision and bias of the lateral estimates, $\tilde{\sigma}_{x, y}$ and $\tilde{B}_{x, y}$, ranged from $1.6 \%$ to $3.6 \%$ and $-4.5 \%$ to $-0.6 \%$. For the axial estimates at $\alpha=90^{\circ}, \tilde{\sigma}_{z}$ and $\tilde{B}_{z}$ were found in the range of 0.3 to $0.5 \%$ and -0.1 to $0.0 \%$. The axial bias $\tilde{B}_{z}$ increased slightly when decreasing $\alpha$ from $90^{\circ}$ to $60^{\circ}$ and the depth from $20 \mathrm{~mm}$ to $30 \mathrm{~mm}$.

The SD $\tilde{\sigma}_{x, y}$ for the transverse estimates increased slightly when lowering $\alpha$ from $90^{\circ}$ to $75^{\circ}$; the average increase was $\Delta \tilde{\sigma}_{x, y}=0.6 \%$ at depth $=20 \mathrm{~mm}$, and $\Delta \tilde{\sigma}_{x, y}=0.78 \%$ at $30 \mathrm{~mm}$. Lowering $\alpha$ from $75^{\circ}$ to $60^{\circ}$ increased $\tilde{\sigma}_{x, y}$ by $1.7 \%$ at $20 \mathrm{~mm}$ and $2.48 \%$ at $30 \mathrm{~mm}$. This demonstrates that positioning of the probe in relation to the flow has an influence on the velocity estimator, especially with $\alpha$ and depth, as a larger increase in bias was observed with larger depths, and with large $\alpha$.

Correlations per estimate $\left(C_{\text {est }}\right)$ : The $C_{\text {est }}$ parameter denotes the number of correlated HRV pairs per velocity estimate. It can be seen from Fig. 6 (b) that the precision is roughly proportional to $1 / \sqrt{C_{\text {est }}}$. The best results were found for the highest $C_{e s t}$; here $\left(\tilde{B_{x}}, \tilde{B_{y}}, \tilde{B}_{z}\right)=-(3.0,3.2,3.3) \%$ and $\left(\tilde{\sigma}_{x}, \tilde{\sigma}_{y}, \tilde{\sigma}_{z}\right)=(1.8,1.3,0.3) \%$. The bias was not affected by $C_{e s t}$. For all components the SDs were below $6 \%$ for $C_{e s t}=16$ and below $7 \%$ for $C_{e s t}=4$, indicating that very fast imaging can be attained with good precision.

Pulse repetition frequency $\left(f_{\text {prf }}\right)$ : $f_{\text {prf }}$ was varied from 1 to $10 \mathrm{kHz}$, for three different values of $\alpha$ at $(60,75,90)^{\circ}$. Fig. 6(c) shows that the bias of the lateral estimates at $\alpha \neq 90^{\circ}$ are affected for $f_{\text {prf }}$ values below $5 \mathrm{kHz}$. The sudden spike in bias at $2 \mathrm{kHz}$ for the lateral ( $x$ and $y$ ) estimates indicates that low values $f_{p r f}$ should be avoided for the transverse components at this velocity. The performance of the axial estimator remains constant throughout but decreases at $\alpha=60^{\circ}$ for $f_{\text {prf }}=1 \mathrm{kHz}$. At $\alpha=90^{\circ}$, the estimator performs well for all values of $f_{p r f}$. The SDs remain below $4 \%$ for all velocity components and all values of $f_{p r f}$. This shows that the estimator performs well if the flow is predominantly in $x, y$-directions, but when axial flow is present (decreasing $\alpha$ ) then the performance suffers. This suggests that the estimator is sensitive to axial motion.

\section{B. Interleaved vs. non-interleaved using RCAs}

Fig. 7 shows velocity profiles estimated from simulations for a) lateral flow with $v_{\text {peak }}$ varied from 10 to $400 \mathrm{~cm} / \mathrm{s}$, and b) axial flow with $v_{\text {peak }}$ varied from 10 to $75 \mathrm{~cm} / \mathrm{s}$. A summary of the relative bias and relative SD is shown in Fig. 8. For lateral flow, both sequences attain a low SD for velocities below $1 \mathrm{~m} / \mathrm{s}$. For the non-interleaved sequence, the SD increases rapidly when the velocity exceeds $1 \mathrm{~m} / \mathrm{s}$ yielding unreliable profiles. For the interleaved sequence, the SD remains low, but the bias increases with the velocity.

\section{Measurement results}

1) Flow-rig: From the flow rig measurement, the parabolic flow profiles were extracted and compared to the true velocity profile of the three components using $\tilde{B}$ and $\tilde{\sigma}$. Fig. 9 shows a subset of the profiles. The top row shows a beam-to-flow angle of $90^{\circ}$, decreasing to $75^{\circ}$ for the center row, down to $60^{\circ}$ in the last row. Here, $\beta=45^{\circ}$ for all and $v_{\text {peak }}=25 \mathrm{~cm} / \mathrm{s}$. As in the simulations, an increase in axial flow $\left(v_{z}\right.$, when $\alpha$ increases) results in a bias increase. For $\alpha=60^{\circ}$ (bottom row) the estimator suffers, seen by the deviation from the true profile close to the edge of the tube. However, notice how 

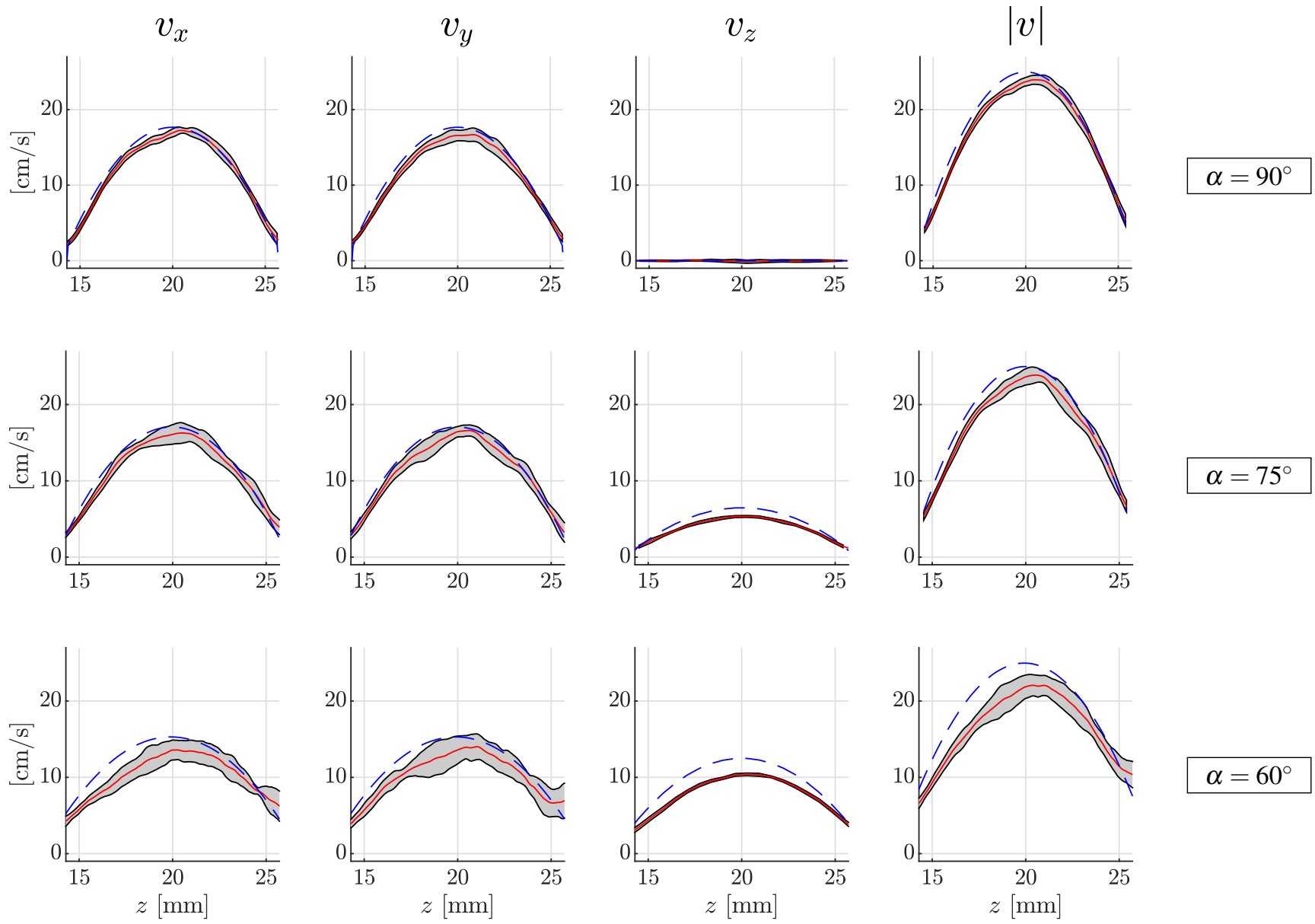

Fig. 5: Estimated center profiles from simulated data for $\alpha=(60,75,90)^{\circ}, \beta=45^{\circ}$. Standard settings from Table II were used. All three components are visualized, including the magnitude. The center of the vessel was placed at a depth of $30 \mathrm{~mm}$. The true parabolic profiles are shown in dashed blue and calculated using (12) and (13). The red curve is the estimated mean profile ( $\tilde{v})$, and the gray shaded area shows the standard deviation $(\tilde{\sigma})$. The results for all settings can be found in Fig. 6 .

the SD $(\sigma)$ remains below $7.9 \%$ for all $\alpha$, indicating high precision.

The overall performance of the estimator on measured data is summarized in Fig. 10. For the slower flow setting of $v_{\text {peak }}=10 \mathrm{~cm} / \mathrm{s}$ the largest bias and SDs were $5.8 \%$ and $6.8 \%$ except for $\alpha=60^{\circ}, \beta=0$ with an outlier of $15.5 \%$. With the higher velocity of $v_{\text {peak }}=25 \mathrm{~cm} / \mathrm{s}$ and $\beta=45^{\circ}$, the transverse bias $\left(\tilde{B}_{x}, \tilde{B}_{y}\right)$ had a linear increase to an absolute value of $21.6 \%$, and the SD were below $9 \%$ for all three components. This trend is the same as revealed in the simulations, although the increase is larger. Having $\beta=0$ resulted in a higher velocity along $x$ and caused the bias along $x\left(\tilde{B}_{x}\right)$ to increase in magnitude from $-17 \%$ to $-54.2 \%$ due to the de-correlation of the summed data.

2) Pulsating flow: In Fig. 11, two orthogonal B-mode slices are shown, with the corresponding velocity vectors imaged on top in 3-D. The estimated velocity field was sampled at $31 \times 31 \times 41$ voxels, providing estimates in the entire volume as a function of $x, y, z$ and time $t$. Three points have been selected for showing the velocity: a point in the center of the vessel (center), a point closer to the vessel wall (near wall) and one outside the vessel (outside). Each of the three selected points have their velocity components plotted as a function of time in Fig. 12. The flow is pulsating inside the vessel. The highest velocity component is expected to be along $x$, due to the orientation of the vessel in relation to probe. As expected, the flow in the center of vessels is overall higher, compared to the point closer to the vessel wall. The peak velocity at the inlet was measured as a function of time and divided into sections. The length of each section corresponded to a single flow pump cycle of $840 \mathrm{~ms}$, and 10 ensembles were collected. The mean and standard deviation were calculated across the ensembles as seen in Fig. 12 d). The relative standard deviation of $10.9 \%$ was calculated across the profile with (19), and mean and standard deviation was calculated with (16) and (17).

Two videos are presented with the paper. Video 1 contains the full volumetric 4-D $(x, y, z, t)$ display of the measurement, including the three selected points. Here, $C_{e s t}$ was 10 and a 

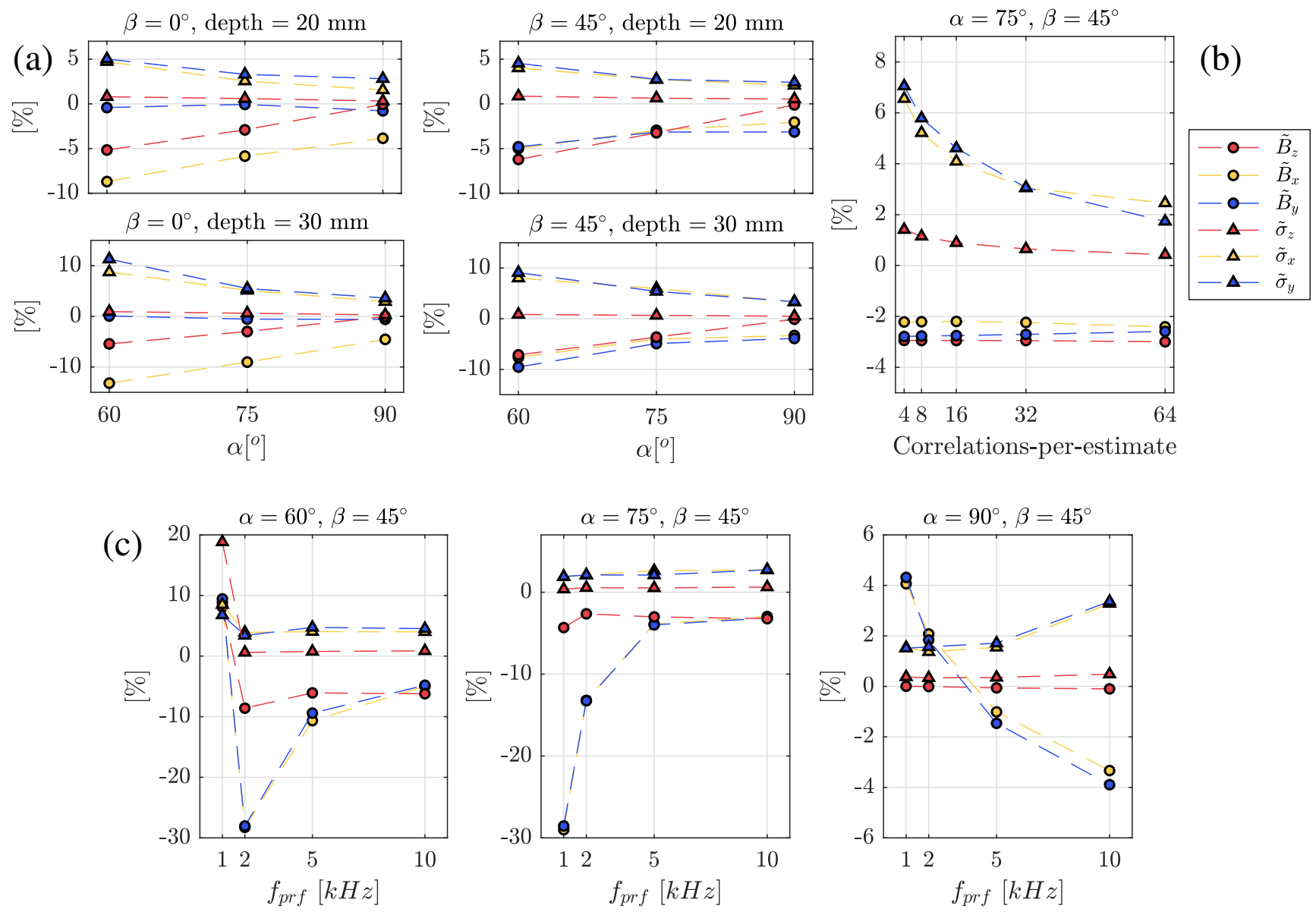

Fig. 6: Summarized results of the parameter study. Four panels in (a) show bias $\left(\tilde{B}_{x, y, z}\right)$ and SD ( $\left.\tilde{\sigma}_{x, y, z}\right)$ for the two rotation angles $(\alpha, \beta)$ and two depths $(20 \mathrm{~mm}, 30 \mathrm{~mm})$. (b) Shows how the number of correlations per estimate affects bias and SD. (c) Three plots showing the effect of pulse repetition frequency on bias and SD. All simulations were made with a velocity of $25 \mathrm{~cm} / \mathrm{s}$. Please note that the standard settings of Table II were used, highlighted in bold.

sliding window was employed to maintain the high volume rate. The second video shows the volumetric velocity data from several positions as a function of time to show the pulsation.

\section{DISCUSSION}

The sequence has been investigated using simulations and measurements. Phantom measurements revealed a penetration depth of $14 \mathrm{~cm}$ in the rectilinear volume beneath the probe surface for both sequences. This roughly corresponds to the penetration depth for normal $3 \mathrm{MHz}$ abdominal probes. This is a benefit of utilizing the entire length of the elements in transmit, without connecting to each matrix element separately. Focusing is performed in both transmit and receive from having several emissions. The sequence utilizes this, so only 32 elements have to be controlled in transmit and 62 in receive, while still capturing data from the entire volume using SA.

\section{A. Performance}

Simulations demonstrated that the velocity estimator could capture all three velocity components with a low standard deviation and bias for $f_{p r f}=10 \mathrm{kHz}$ and $v_{\text {peak }}=25 \mathrm{~cm} / \mathrm{s}$.
Lowering $f_{\text {prf }}$ to $2 \mathrm{kHz}$ revealed a larger bias increase of up to $30 \%$ for the transverse components, likely due to decorrelation. The largest bias was seen at $60^{\circ}$. The estimator performs well at $\alpha=90^{\circ}$ for all tested values of $f_{\text {prf }}$, however, the performance decreased for $\alpha \neq 90^{\circ}$, which indicates that higher axial motion degrades the accuracy of the estimator. The directional TO method is designed to filter out the axial oscillation, however the larger $\tilde{B}_{z}$ with lower $\alpha$ suggest that this is not fully attained, and this influences the transverse estimates. This can be attributed to the estimator design, which assumes monochromatic oscillations both axially and laterally, which is not true for pulsed fields, or a result of a larger dephasing of consecutive LRVs during SA summation with large velocities.

The measurement setup suffered from the probe being a prototype, as elements were failing ( 8 dead rows). Beside this, the transducer handle was slightly leaky, and a latex cover was used to avoid damage to the electronics. To lower the effect of reverberation from the side of the water tank, a $f_{p r f}$ of $2 \mathrm{kHz}$ was employed for the flow rig measurements. All of these factors adversely affect the measured data, and thereby the performance of the estimator. Nevertheless, a similar 

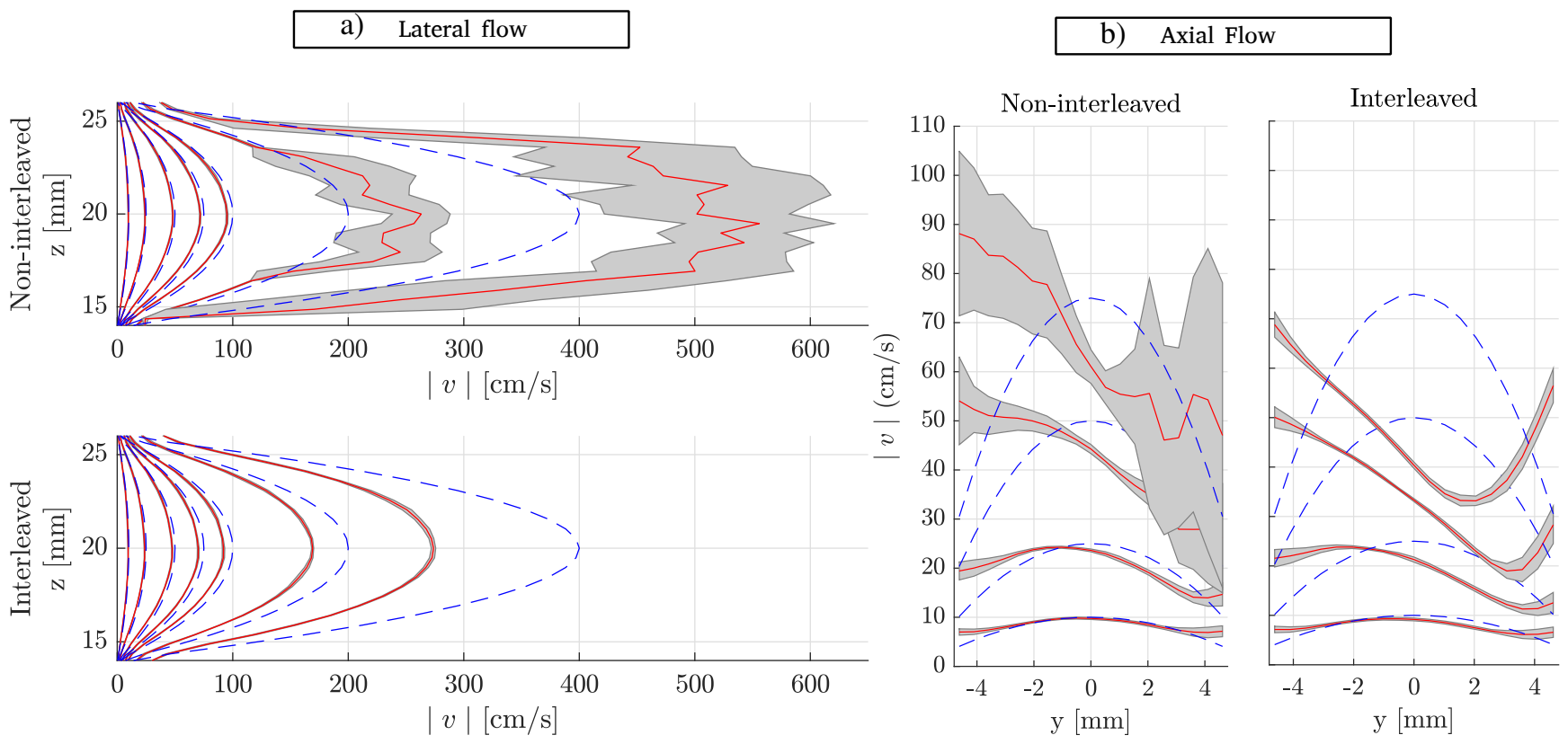

Fig. 7: Simulation results of the interleaved vs. non-interleaved sequence for pure lateral flow (a), and pure axial flow (b). Red shows the estimated mean ( $\tilde{v})$, gray is $\pm \sigma$, and the blue dotted line shows the true profile $\left(v_{\text {true }}\right)$.
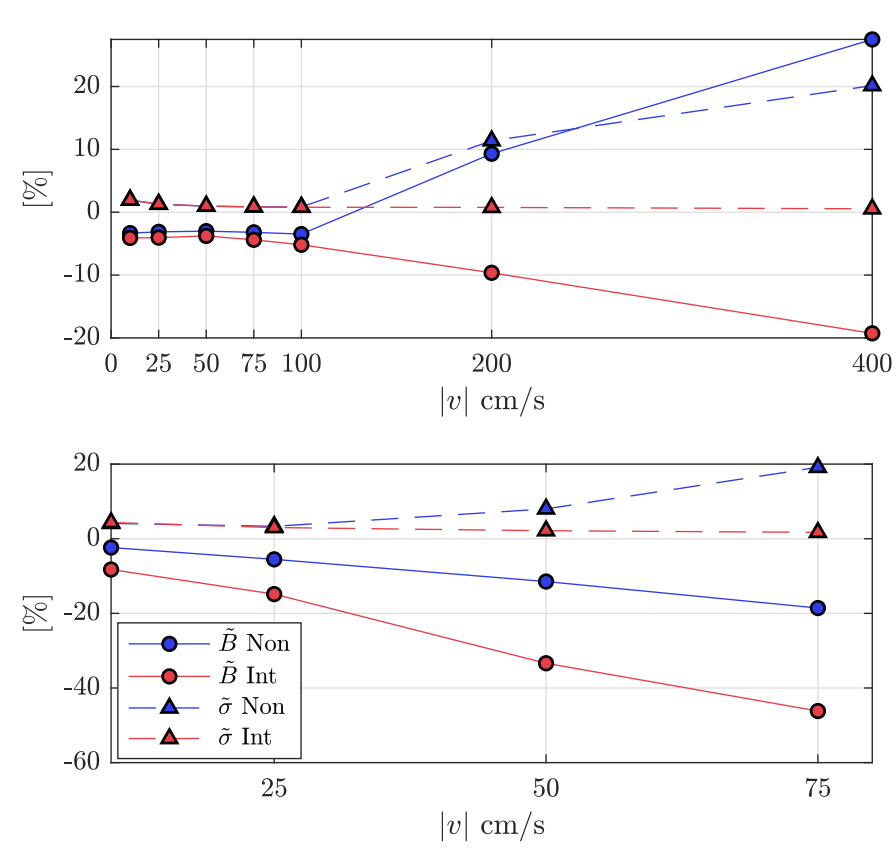

Fig. 8: Performance of the interleaved (red) vs. non-interleaved (blue) sequences. Top plot shows performance in the lateral direction, and the bottom plot shows axial velocity estimates.

trend as in the simulations were observed. The estimator performed well when flow was predominantly in the transverse direction. For the low velocity setting of $v_{\text {peak }}=10 \mathrm{~cm} / \mathrm{s}$, the relative bias and standard deviation was below $7 \%$ for all angle combinations. This is seen in Fig. 10a, except for $(\alpha, \beta)=\left(60^{\circ}, 0^{\circ}\right)$, where the highest axial component and highest $v_{x}$ component is expected. The large drop in $\tilde{B}_{x}$ shows the estimator is challenged. For the faster peak flow of 25 $\mathrm{cm} / \mathrm{s}$ the bias seems to increase with decreasing $\alpha$, similar to the simulations. This was observed for $\beta=45^{\circ}$. Rotating the probe to $\beta=0^{\circ}$ increases the $v_{x}$ component and the estimator degrades for $\alpha \neq 90$. A peak velocity in the range of 10 $\mathrm{cm} / \mathrm{s}$ to $25 \mathrm{~cm} / \mathrm{s}$ seems to be the limit pulsing at $2 \mathrm{kHz}$ in the measurements. Utilizing $10 \mathrm{kHz}$ would then result in a maximum detectable velocity in the range of $50-125 \mathrm{~cm} / \mathrm{s}$.

\section{B. Interleaved vs non-interleaved}

The interleaved sequence increases the summation time by a factor of two. This will for fast moving point targets cause a de-phasing in the summation of LRVs, which is twice compared to the non-interleaved sequence. A longer summation time has been shown to increase bias [51], [52] when employing SA imaging for flow estimation. This explains why, at lower flow velocities, the bias of the noninterleaved sequence is lower, as indicated in Fig. 7 and Fig. 8. However, the longer summation time associated with the interleaved sequence translate to a greater spatio-temporal overlap between moving points observed in two consecutive HRVs. This allows the DTO to estimate higher velocities with higher precision, although with lower accuracy. Since the transverse wavelength $\left(\lambda_{x}\right)$ given by (5) is larger than the axial wavelength, the effect is less dominant with purely lateral flow.

\section{Limitations and mitigation}

The proposed velocity estimation contains certain limitations; especially with frame rate, with high axial velocity, underestimation of flow, and a limited field of view. The 

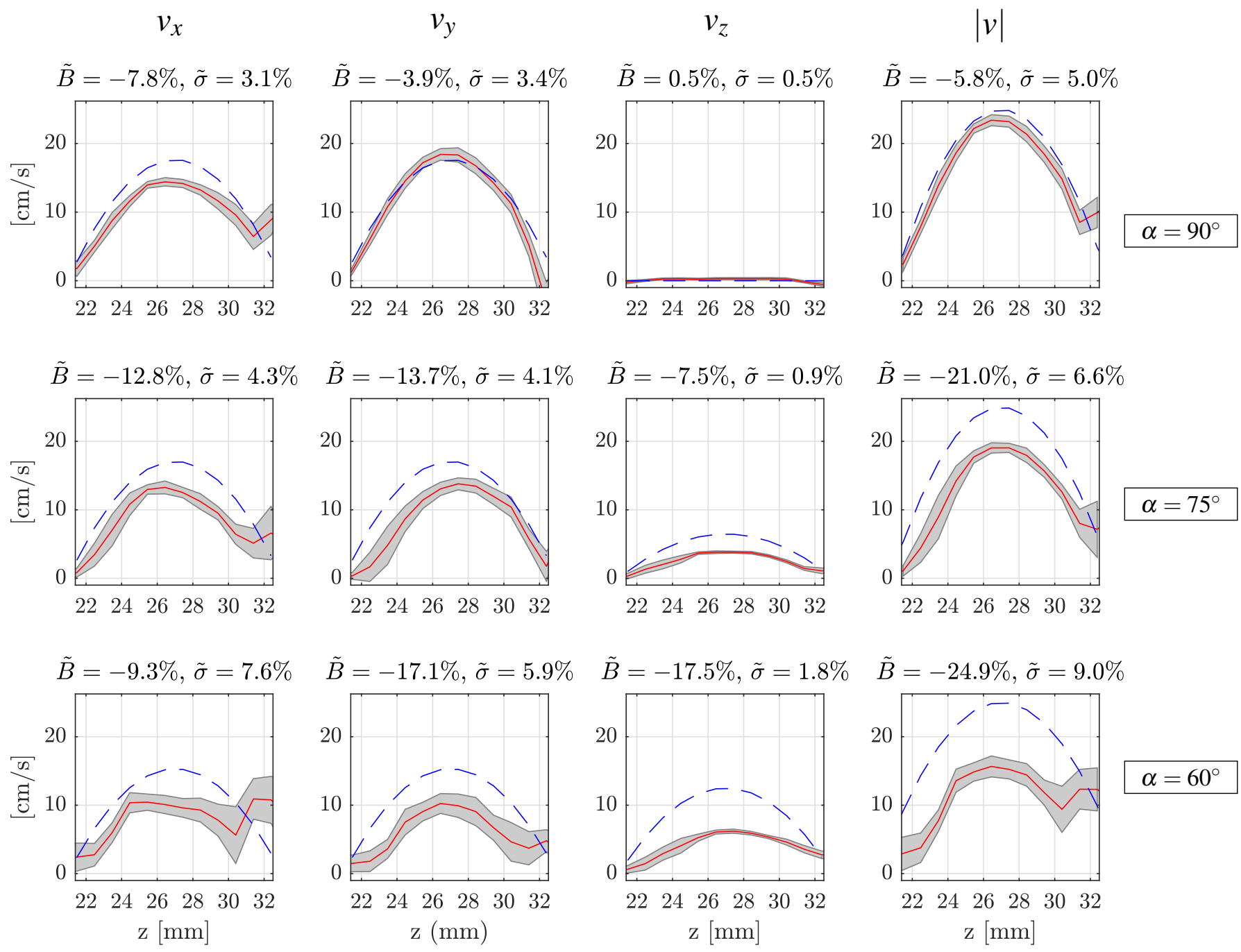

Fig. 9: Estimated velocity profiles of all three components from the constant flow measurements for $v_{\text {peak }}=25 \mathrm{~cm} / \mathrm{s}$ and $\beta=$ $45^{\circ}$ for various angles $\alpha$. The dashed blue line is the true velocity, the red line is the estimated mean velocity profile, and the shaded gray area indicates one SD. The mean estimated bias $\tilde{B}$ and standard deviation $\tilde{\sigma}$ are shown in the titles.

one way focusing of RCAs [22] requires 8 emissions per HRV to achieve focusing along the transmit direction. This however, has the consequence of lowering the effective $f_{\text {prf }}$. The interleaved sequence help maintain precision (low SD) for larger velocities, although it suffers in terms of accuracy (high bias). Below follows a brief discussion on each of the limitations separately, although the effects cannot directly be isolated and has to be considered in relation to each others.

1) Frame rate: A major limiting factor in achieving full 4-D volumetric vector flow imaging in a 3-D volume with B-mode guidance has been the frame rate. Optimized SA imaging (also referred to as coherent compounding imaging) usually requires 22-32 emissions to achieve a high contrast [42], [43]. In measurements the $f_{p r f}$ was lowered to $2 \mathrm{kHz}$ to avoid reverberations.

However, the $f_{p r f}$ utilized in the simulations $(10 \mathrm{kHz})$ would lead to a volume rate of $250 \mathrm{~Hz}$ for flow and $125 \mathrm{~Hz}$ for Bmode if $C_{e s t}=1$. A more realistic setup would utilize $C_{e s t}=8$ to 16 . Employing $C_{\text {est }}=10$ as in the measurements, would limit the volume rate to $25 \mathrm{~Hz}$ for independent estimates. However, employing a sliding window approach would increase this, although this would introduce slight smoothing of the velocity. To mitigate this a larger $f_{p r f}$ could be employed, as done in [35]. An imaging depth of $4 \mathrm{~cm}$ would be sufficient in carotid imaging for most patients, resulting in an $f_{p r f}$ of $19 \mathrm{kHz}$, and a volume rate above $45 \mathrm{~Hz}$.

2) High axial velocity component: As uncovered in section VI-B, a high axial velocity will cause a phase shift of the individual LRVs. This is believed to cause the spatial skewing of the profiles in Fig. 7 b), with purely axial flow. Two vessels placed at the same depth could potentially affect each other, when a strong axial velocity is present, although this remains to be investigated.

Comparing the magnitude profiles in Fig. 5 for an $f_{\text {prf }}$ of $10 \mathrm{kHz}$, to Fig. 9 with $2 \mathrm{kHz}$, seems to substantiate that a decorrelation of the individual LRVs is a potential cause of the bias increase with increasing axial velocity. The bias increase with decreasing $\alpha$ is more severe due to the higher 

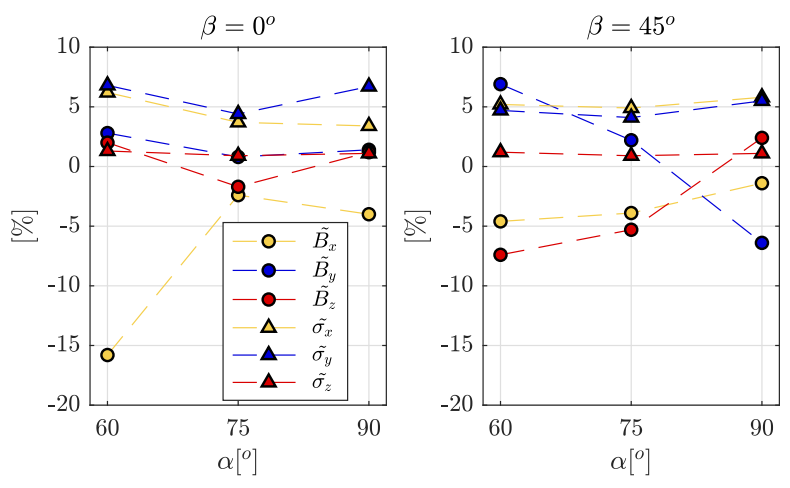

(a) $v_{\text {peak }}=10 \mathrm{~cm} / \mathrm{s}$.
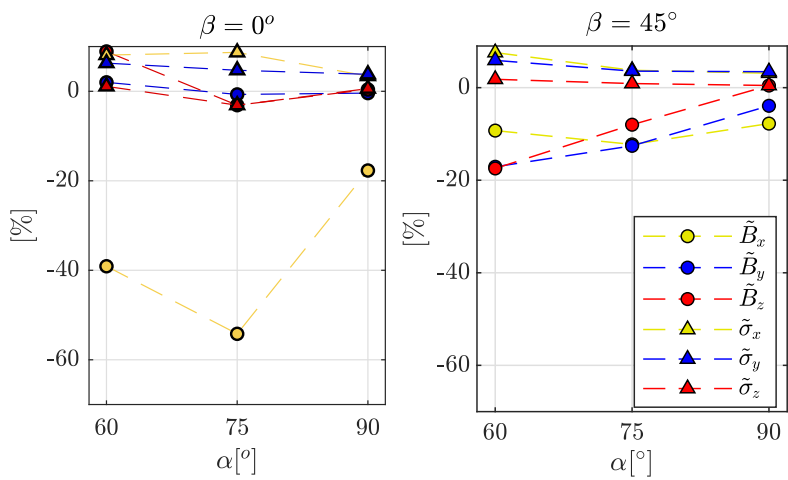

(b) $v_{\text {peak }}=25 \mathrm{~cm} / \mathrm{s}$.

Fig. 10: Summary of the performance for $\beta=0^{\circ}$ and $\beta=$ $45^{\circ}$ for $10 \mathrm{~cm} / \mathrm{s}$ in (a) and for $25 \mathrm{~cm} / \mathrm{s}$ in (b). $\left(\tilde{B}_{x}, \tilde{B}_{y}, \tilde{B}_{z}\right)$ is the relative bias, and $\left(\sigma_{x}, \sigma_{y}, \sigma_{z}\right)$ are the relative standard deviations for all three estimated components. Acquired with imaging parameters given in I.

temporal spacing associated with the lower $f_{p r f}$. This is, however, not unique to the RCA setup, but might be more pronounced due to the one way focusing [53] associated with these arrays. However, techniques such as motion estimation and correction might improve the estimator and increase the maximum detectable velocity [51], [52], and compensate the de-phasing. Often these techniques benefit from an initial guess of the velocity, which avoids unnecessary computations by limiting the search region, and improves the stability of the estimator.

3) Underestimation of flow: As uncovered by the measurements and simulations, the velocity limit depends on $\alpha$ and $\beta$. However, the operator could mitigate this by a rotation and translation of the probe. When $\beta=45$, the transverse velocity is divided into both $x$ and $y$ components as indicated by (13). This in turns lowers the required maximum velocity along both $x$ and $y$. A larger bias is observed for larger velocities, and the clinical relevance remains to be investigated. The TO method has previously been associated with an overall $10 \%$ underestimation of flow compared to MR Angiography [54]. However, when high accuracy is required, a more conventional estimation method could be employed as demonstrated in [32], where the high volume rate can be substituted for higher accuracy, or with high frame rate but in a smaller volume.

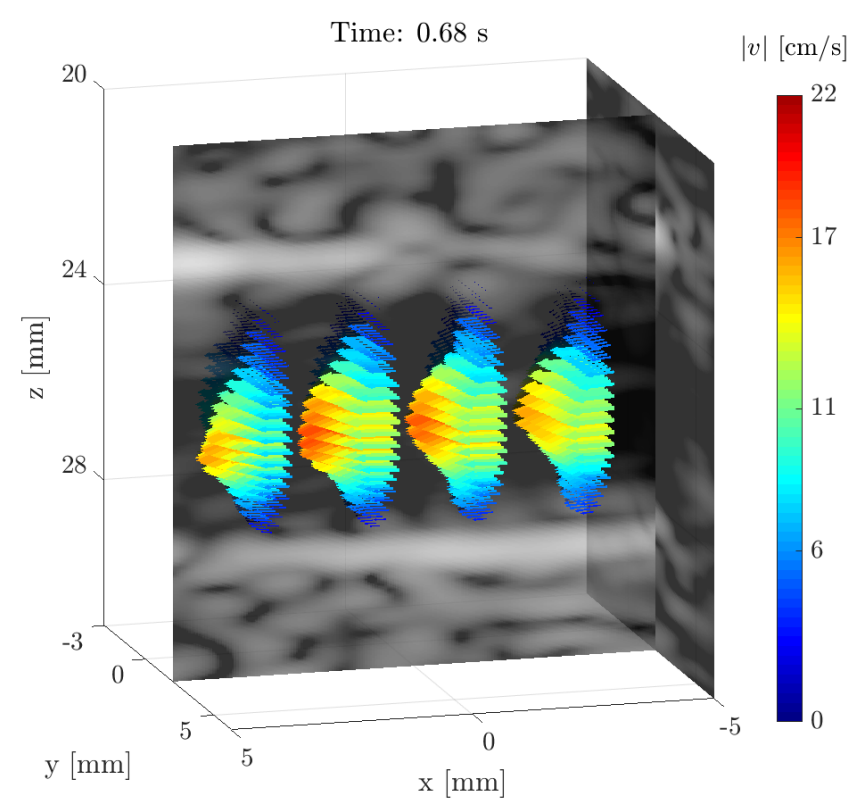

Fig. 11: Pulsating flow in a tissue mimicking phantom. The Bmode planes can be selected in the entire volume. The dynamic range is $40 \mathrm{~dB}$ for the $\mathrm{B}$-mode. The flow is visualized using arrows, where color shows magnitude and arrows symbolize velocity direction. The accompanying videos show the volume over time for different orientations with and without the measurements indicated in Fig. 12.
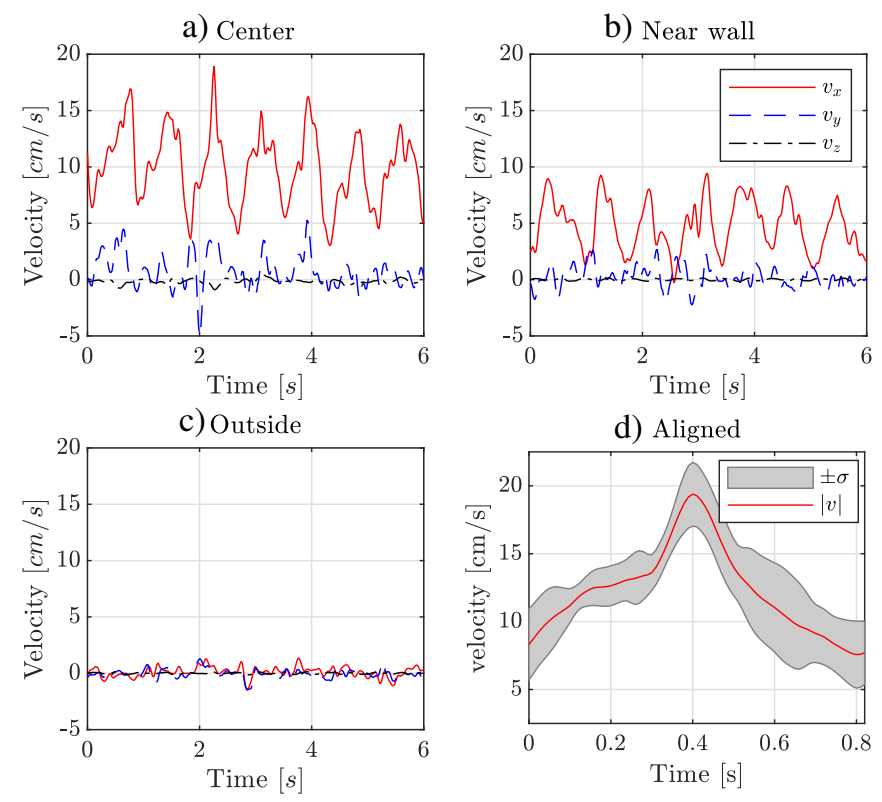

Fig. 12: a) shows all three velocity components $v_{x}, v_{y}, v_{z}$ (red, blue, black) for a point in the vessel center. b) shows the components at a point placed near the vessel wall. c) for a point placed outside the vessel. d) shows peak velocity at inlet, the mean of the peak velocity in 10 aligned pulse cycles in red and gray is \pm one standard deviation, from the data in a). 
4) Depth range and Field of View: The imaging sequence is capable of reaching depths of $14 \mathrm{~cm}$ in terms of SNR. However, the depth range of the velocity estimator is limited, due to the limited side-length, which limits the DP apodization width (see Fig. 2). This is substantiated by the results in Fig. 6 (a), where an increase in depth $(10 \mathrm{~mm})$ gave an overall decrease in performance. This is directly related to (5), where the width of the physical aperture limits $d_{x}$, and an increase of lateral wavelength is observed as a function of depth. At $14 \mathrm{~cm}$ the lateral wavelength will be close to the side-length of this array, negatively impacting any method that attempts to estimate lateral motion.

The higher penetration of $14 \mathrm{~cm}$ can, however, be traded for a higher transmit center frequency, which in turn translates to a higher resolution, expected to improve the estimator. The side-length of RCAs also scales linearly with the number of elements, allowing for much larger apertures to be manufactured without a high complexity in terms of interconnections. The current sequence has been validated down to $30 \mathrm{~mm}$. The field of view depends on the spatial overlap of the transmitted waves, along with spatial sampling of VSLs as dictated by the effective source pitch. The transmitted waves have $100 \%$ overlap in a 17 by $17 \mathrm{~mm}^{2}$ area, at depth $20-30 \mathrm{~mm}$. The flow sequence is expected to generate grating lobes at $\sin ^{-1}\left(\frac{\lambda}{2 \cdot p_{s}}\right)$ $=25^{\circ}$ in transmit. The peak response of the grating lobes were evaluated to be $10-15 \mathrm{~dB}$ lower than the main lobe peak energy. At $20 \mathrm{~mm}$ depth, the grating lobe is at the edge of FOV from where the array's receive sensitivity is very low, and the asymmetry of the TO degrades the performance of the estimator. In receive $\lambda / 2$ pitch ensure no grating lobes which is where the TO apodization is applied, and flow is estimated. The flip in transmit direction (rows/columns) ensures that no grating lobes occur along the direction of the flow estimation. Quantifying the effect of grating lobes in terms of flow estimation is difficult, but suppression of these is believed to improve the estimator as well, which would require further investigations.

5) Stationary echo removal: The stationary echo removal employed here is a simple mean subtraction, and a clinical implementation probably requires a more advanced filter. The data sequences $\mathrm{HRV}_{R}^{a}$ and $\mathrm{HRV}_{R}^{a}$ are uniformly sampled, and if processed separately, then any echo cancelling approach can be employed. These include IIR, FIR [21], or more advanced filters such as Singular Value Decomposition [55]. However, their effectiveness in relation to this specific sequence remains to be investigated.

\section{RCAs and ultrasonic TVI}

The method presented here, although with certain limitations, is capable of estimating the transverse components consistently and shows the potential for the use of RCA on systems no more complex than current commercial scanners. The main limitation to overcome for a broad clinical application is the axial de-correlation problem already discussed. When flow is predominantly in transverse direction the flow can be estimated consistently with a slight bias.

The velocity of the pulsating flow was estimated in the entire volume, and the peak velocity at the inlet was likewise estimated with a relative standard deviation of $10.9 \%$. The data is continuous everywhere in the volume and quantitative measures for peak and mean velocity along with volume flow and other derived quantities can be obtained. The continuous data also makes it possible to use the sequence for functional ultrasound imaging [35], [56] and super resolution imaging [57]-[63] in 3-D [64], [65]. The observation time can be made as long as necessary, so there is essentially no lower limit on the velocity [66]. It should also be noted that all processing is done retrospectively after data collection, so a measurement can be processed for both high- and low velocity estimation.

This sequence only requires 124 channels to be connected to the probe. In transmit only 32 elements must be controlled, and in receive only 62 channels were sampled per each emission. While capable of utilizing the entire surface area of a $62^{2}$ matrix array, the hardware requirements are low. This propagates directly to the processing as well, as only 62 channels and not $62^{2}$ have to be processed per emission. Most scanners can sample at least 128 channels simultaneously. This means that TVI in an entire volume can be implemented on a modified commercial scanner using only 124 channels. GPU beamforming with state-of-the-art graphic cards are capable of performing real-time RCA processing for crossing B-mode planes, as the low channel count allows for fast input to the GPU [49].

\section{CONCLUSION}

TVI (4-D volumetric vector flow imaging) using a RCA was demonstrated. The method was evaluated through simulations and measurements with the use of a novel sequence design incorporating synthetic aperture techniques and interleaved emissions to provide continuous data in the entire 3-D volume. The method can provide the transverse components of the vector field utilizing directional transverse oscillations to estimate flow in a volume. This is attained with only 62 channels in receive and 32 in transmit for full TVI. The SD of the estimator was low for all three components, due to the new interleaved volumetric RCA SA sequence. A bias increase was observed with increasing velocity, an effect more pronounced for the interleaved method, but the precision of the proposed method was high. A maximum peak velocity of 50 to $125 \mathrm{~cm} / \mathrm{s}$ should be within reach, while having volumetric B-mode guidance at the same time at high volume rates. The method is then suited for measuring on the carotid artery and other shallow vessels, where the flow is parallel to the transducer surface. The RCA scheme makes linear scaling of the resolution with the number of elements possible, and the high penetration depth can be employed in attaining even better B-mode performance and flow estimates.

\section{ACKNOWLEDGEMENT}

This work was financially supported by grant 82-2014-4 from the Danish National Advanced Technology Foundation, by grant 7050-00004B from Innovation Fund Denmark, and from BK Medical, Herlev, Denmark. 


\section{REFERENCES}

[1] S. W. Smith, H. G. Pavy, and O. T. von Ramm, "High speed ultrasound volumetric imaging system - Part I: Transducer design and beam steering," IEEE Trans. Ultrason., Ferroelec., Freq. Contr., vol. 38, pp. 100-108, 1991.

[2] O. T. von Ramm, S. W. Smith, and H. G. Pavy, "High speed ultrasound volumetric imaging system - Part II: Parallel processing and image display," IEEE Trans. Ultrason., Ferroelec., Freq. Contr., vol. 38, pp. 109$115,1991$.

[3] A. Fenster, D. B. Downey, and H. N. Cardinal, "Three-dimensional ultrasound imaging," Phys. Med. Biol., vol. 46, pp. R67-R99, 2001.

[4] A. Harloff, F. Albrecht, J. Spreer, A. F. Stalder, J. Bock, A. Frydrychowicz, J. Schollhorn, A. Hetzel, M. Schumacher, J. Hennig, and M. Markl, "3D blood flow characteristics in the carotid artery bifurcation assessed by flow-sensitive 4D MRI at 3T," Magn. Reson. Med., vol. 61, no. 1, pp. 65-74, 2009.

[5] M. Correia, J. Provost, M. Tanter, and M. Pernot, "4D ultrafast ultrasound flow imaging: in vivo quantification of arterial volumetric flow rate in a single heartbeat," Phys. Med. Biol., vol. 61, no. 23, pp. L48L61, 2016.

[6] M. S. Wigen, S. Fadnes, A. Rodriguez-Molares, T. Bjåstad, M. Eriksen, K. H. Stensæth, A. Støylen, and L. Løvstakken, "4-D intracardiac ultrasound vector flow imaging-reasibility and comparison to phasecontrast MRI," IEEE Trans. Med. Imag., vol. 37, pp. 2619-2629, December 2018

[7] J. Provost, C. Papadacci, C. Demene, J.-L. Gennisson, M. Tanter, and M. Pernot, "3-d ultrafast Doppler imaging applied to the noninvasive mapping of blood vessels in vivo," IEEE Trans. Ultrason., Ferroelec., Freq. Contr., vol. 62, no. 8, pp. 1467-1472, 2015.

[8] C. Papadacci, V. Finel, O. Villemain, G. Goudot, J. Provost, E. Messas, M. Tanter, and M. Pernot, " $4 \mathrm{~d}$ simultaneous tissue and blood flow doppler imaging: revisiting cardiac doppler index with single heart beat 4d ultrafast echocardiography," Phys. Med. Biol., vol. 64, pp. 1-10, 2019.

[9] J. A. Jensen, H. Holten-Lund, R. T. Nilsson, M. Hansen, U. D. Larsen, R. P. Domsten, B. G. Tomov, M. B. Stuart, S. I. Nikolov, M. J. Pihl, Y. Du, J. H. Rasmussen, and M. F. Rasmussen, "SARUS: A synthetic aperture real-time ultrasound system," IEEE Trans. Ultrason., Ferroelec., Freq. Contr., vol. 60, no. 9, pp. 1838-1852, 2013.

[10] R. E. Davidsen, J. A. Jensen, and S. W. Smith, "Two-dimensional random arrays for real time volumetric imaging," Ultrason. Imaging, vol. 16, pp. 143-163, July 1994.

[11] S. S. Brunke and G. R. Lockwood, "Broad-bandwidth radiation patterns of sparse two-dimensional vernier arrays," IEEE Trans. Ultrason., Ferroelec., Freq. Contr., vol. 44, pp. 1101-1109, sep 1997.

[12] J. W. Choe, O. Oralkan, and P. Khuri-Yakub, "Design Optimization for a 2-D sparse Transducer Array for 3-D Ultrasound Imaging," Proc. IEEE Ultrason. Symp., vol. 2010, pp. 1928-1931, 2010.

[13] B. Diarra, M. Robini, P. Tortoli, C. Cachard, and H. Liebgott, "Design of optimal 2-D nongrid sparse arrays for medical ultrasound," IEEE Trans. Biomed. Eng., vol. 60, no. 11, pp. 3093 - 3102, 2013.

[14] A. Austeng and S. Holm, "Sparse 2-D arrays for 3-D phased array imaging - design methods," IEEE Trans. Ultrason., Ferroelec., Freq. Contr., vol. 49, pp. 1073-1086, August 2002.

[15] E. Roux, F. Varray, L. Petrusca, C. Cachard, P. Tortoli, and H. Liebgott, "Experimental 3-D Ultrasound Imaging with 2-D Sparse Arrays Using Focused and Diverging Waves," Scientific Reports, vol. 8, no. 9108, 2018.

[16] C. E. Morton and G. R. Lockwood, "Theoretical assessment of a crossed electrode 2-D array for 3-D imaging," in Proc. IEEE Ultrason. Symp., pp. 968-971, 2003.

[17] C. E. M. Démoré, A. Joyce, K. Wall, and G. Lockwood, "Real-time volume imaging using a crossed electrode array," IEEE Trans. Ultrason., Ferroelec., Freq. Contr., vol. 56, no. 6, pp. 1252-1261, 2009.

[18] J. T. Yen, C. H. Seo, S. I. Awad, and J. S. Jeong, "A dual-layer transducer array for 3-D rectilinear imaging," IEEE Trans. Ultrason., Ferroelec., Freq. Contr., vol. 56, no. 1, pp. 204-212, 2009.

[19] A. Sampaleanu, P. Zhang, A. Kshirsagar, W. Moussa, and R. Zemp, "Top-orthogonal-to-bottom-electrode (TOBE) CMUT arrays for 3-D ultrasound imaging.," IEEE Trans. Ultrason., Ferroelec., Freq. Contr., vol. 61, no. 2, pp. 266-276, 2014.

[20] M. F. Rasmussen, T. L. Christiansen, E. V. Thomsen, and J. A Jensen, "3-D imaging using row-column-addressed arrays with integrated apodization - Part I: Apodization design and line element beamforming," IEEE Trans. Ultrason., Ferroelec., Freq. Contr., vol. 62, no. 5, pp. 947-958, 2015.
[21] S. Bjærum, H. Torp, and K. Kristoffersen, "Clutter filter design for ultrasound color flow imaging," IEEE Trans. Ultrason., Ferroelec., Freq. Contr., vol. 49, pp. 204-209, 2002.

[22] H. Bouzari, M. Engholm, S. I. Nikolov, M. B. Stuart, E. V. Thomsen, and J. A. Jensen, "Imaging performance for two row-column arrays," IEEE Trans. Ultrason., Ferroelec., Freq. Contr., vol. 66, no. 7, pp. 1209-1221, 2019.

[23] J. H. Rasmussen, M. C. Hemmsen, S. S. Madsen, P. M. Hansen, M. B. Nielsen, and J. A. Jensen, "Preliminary study of synthetic aperture tissue harmonic imaging on in-vivo data," in Proc. SPIE Med. Imag., vol. 8675, pp. 1-10, 2013.

[24] M. Karaman, P. C. Li, and M. O'Donnell, "Synthetic aperture imaging for small scale systems," IEEE Trans. Ultrason., Ferroelec., Freq. Contr., vol. 42, pp. 429-442, 1995

[25] M. Tanter, J. Bercoff, L. Sandrin, and M. Fink, "Ultrafast compound imaging for 2-D motion vector estimation: application to transient elastography," IEEE Trans. Ultrason., Ferroelec., Freq. Contr., vol. 49, pp. 1363-1374, 2002

[26] J. A. Jensen, S. Nikolov, K. L. Gammelmark, and M. H. Pedersen, "Synthetic aperture ultrasound imaging," Ultrasonics, vol. 44, pp. e5e15, 2006.

[27] J. Sauvage, M. Flesch, G. Ferin, A. Nguyen-Dinh, J. Poree, M. Tanter, M. Pernot, and T. Deffieux, "A large aperture row column addressed probe for in vivo $4 \mathrm{~d}$ ultrafast doppler ultrasound imaging," Phys. Med. Biol., vol. 63, pp. 1-12, 2018.

[28] B. Dunmire, K. W. Beach, K.-H. Labs., M. Plett, and D. E. Strandness, "Cross-beam vector Doppler ultrasound for angle independent velocity measurements," Ultrasound Med. Biol., vol. 26, pp. 1213-1235, 2000.

[29] C. Poelma, "Ultrasound imaging velocimetry: a review," Experiments in Fluids, vol. 58, no. 1, pp. 1-28, 2017.

[30] L. N. Bohs, B. H. Friemal, B. A. McDermott, and G. E. Trahey, "A realtime system for quantifying and displaying two-dimensional velocities using ultrasound," Ultrasound Med. Biol., vol. 19, pp. 751-761, 1993.

[31] J. A. Jensen and P. Munk, "A new method for estimation of velocity vectors," IEEE Trans. Ultrason., Ferroelec., Freq. Contr., vol. 45, no. 3, pp. 837-851, 1998.

[32] S. Holbek, T. L. Christiansen, M. B. Stuart, C. Beers, E. V. Thomsen, and J. A. Jensen, "3-D vector flow estimation with row-column addressed arrays," IEEE Trans. Ultrason., Ferroelec., Freq. Contr., vol. 63, no. 11, pp. 1799-1814, 2016.

[33] S. Holbek, M. B. Stuart, and J. A. Jensen, "Volumetric 3-D vector flow measurements using a 62+62 row-column addressed array," in Proc. IEEE Ultrason. Symp., pp. 1-4, 2017.

[34] S. I. Nikolov and J. A. Jensen, "In-vivo synthetic aperture flow imaging in medical ultrasound," IEEE Trans. Ultrason., Ferroelec., Freq. Contr. vol. 50, no. 7, pp. 848-856, 2003.

[35] J. Sauvage, J. P. e, C. Rabut, G. Férin, M. Flesch, B. Rosinski, A. Nguyen-Dinh, M. Tanter, M. Pernot, and T. Deffieux, "4D Functional imaging of the rat brain using a large aperture row-column array," IEEE Trans. Med. Imag., pp. 1928-1931, 2019.

[36] M. Engholm, H. Bouzari, T. L. Christiansen, C. Beers, J. P. Bagge, L. N. Moesner, S. E. Diederichsen, M. B. Stuart, J. A. Jensen, and E. V. Thomsen, "Probe development of CMUT and PZT row-columnaddressed 2-D arrays," Sens. Actuators A: Phys., vol. 273, pp. 121-133, 2018.

[37] J. A. Jensen, "Estimation of high velocities in synthetic aperture imaging: I: Theory," IEEE Trans. Ultrason., Ferroelec., Freq. Contr., vol. 66 , no. 6, pp. 1024-1031, 2019.

[38] J. A. Jensen, "Estimation of high velocities in synthetic aperture imaging: II: Experimental investigation," IEEE Trans. Ultrason., Ferroelec., Freq. Contr., vol. 66, no. 6, pp. 1032-1038, 2019.

[39] M. Schou, L. T. Jørgensen, M. B. Stuart, M. S. Traberg, B. G. Tomov, and J. A. Jensen, "Full volumetric 3-D vector flow imaging using a 62+62 row-column array," in Proc. IEEE Ultrason. Symp., pp. 1-4, 2019.

[40] J. A. Jensen, "Field: A program for simulating ultrasound systems," Med. Biol. Eng. Comp., vol. 10th Nordic-Baltic Conference on Biomedical Imaging, Vol. 4, Supplement 1, Part 1, pp. 351-353, 1996.

[41] J. A. Jensen and N. B. Svendsen, "Calculation of pressure fields from arbitrarily shaped, apodized, and excited ultrasound transducers," IEEE Trans. Ultrason., Ferroelec., Freq. Contr., vol. 39, no. 2, pp. 262-267, 1992.

[42] R. Moshavegh, J. Jensen, C. A. Villagomez-Hoyos, M. B. Stuart, M. C. Hemmsen, and J. A. Jensen, "Optimization of synthetic aperture image quality," in Proc. SPIE Med. Imag., vol. 9790, pp. 97900Z-97900Z-9, 2016. 
[43] J. Jensen, M. B. Stuart, and J. A. Jensen, "Optimized plane wave imaging for fast and high-quality ultrasound imaging," IEEE Trans. Ultrason., Ferroelec., Freq. Contr, vol. 63, no. 11, pp. 1922-1934, 2016.

[44] M. Flesch, M. Pernot, J. Provost, G. Ferin, A. Nguyen-Dinh, M. Tanter, and T. Deffieux, "4D in vivo ultrafast ultrasound imaging using a rowcolumn addressed matrix and coherently-compounded orthogonal plane waves," Phys. Med. Biol., vol. 62, pp. 4571-4588, 2017.

[45] S. G. Foster, A pulsed ultrasonic flowmeter employing time domain methods. PhD thesis, Dept. Elec. Eng., University of Illinois, Urbana, Ill., 1985.

[46] J. A. Jensen, "A multi-threaded version of Field II," in Proc. IEEE Ultrason. Symp., pp. 2229-2232, IEEE, 2014.

[47] S. S. M. Lai, B. Y. S. Yiu, A. K. K. Poon, and A. C. H. Yu, "Design of anthropomorphic flow phantoms based on rapid prototyping of compliant vessel geometries," Ultrasound Med. Biol., vol. 39, no. 9, pp. 16541664,2013

[48] J. B. Olesen, C. A. Villagómez Hoyos, N. D. Møller, C. Ewertsen, K. L. Hansen, M. B. Nielsen, B. Bech, L. Lönn, M. S. Traberg, and J. A. Jensen, "Non-invasive estimation of pressure changes using 2-D vector velocity ultrasound: An experimental study with in-vivo examples," IEEE Trans. Ultrason., Ferroelec., Freq. Contr., vol. 65, no. 5, pp. 709719, 2018.

[49] M. B. Stuart, M. Schou, and J. A. Jensen, "Row-column beamforming with dynamic apodizations on a GPU," in Proc. SPIE Med. Imag., pp. 17, 2019. Paper number 10955-20.

[50] M. J. Pihl, J. Marcher, and J. A. Jensen, "Phased-array vector velocity estimation using transverse oscillations," IEEE Trans. Ultrason., Ferroelec., Freq. Contr, vol. 59, no. 12, pp. 2662-2675, 2012.

[51] N. Oddershede and J. A. Jensen, "Effects influencing focusing in synthetic aperture vector flow imaging," IEEE Trans. Ultrason., Ferroelec., Freq. Contr., vol. 54, no. 9, pp. 1811-1825, 2007.

[52] I. K. Ekroll, M. M. Voormolen, O. K.-V. Standal, J. M. Rau, and L. Løvstakken, "Coherent Compounding in Doppler Imaging," IEEE Trans. Ultrason., Ferroelec., Freq. Contr., vol. 62, no. 9, 2015.

[53] H. Bouzari, M. Engholm, S. I. Nikolov, M. B. Stuart, E. V. Thomsen, and J. A. Jensen, "Imaging performance for two row-column arrays," IEEE Trans. Ultrason., Ferroelec., Freq. Contr., vol. 66, no. 7, pp. 1209-1221, 2019.

[54] K. L. Hansen, J. Udesen, C. Thomsen, J. A. Jensen, and M. B. Nielsen, "In vivo validation of a blood vector velocity estimator with MR angiography," IEEE Trans. Ultrason., Ferroelec., Freq. Contr., vol. 56, no. 1, pp. 91-100, 2009.

[55] C. Demene, T. Deffieux, M. Pernot, B.-F. Osmanski, V. Biran, J.-L. Gennisson, L.-A. Sieu, A. Bergel, S. Franqui, J.-M. Correas, I. Cohen, O. Baud, and M. Tanter, "Spatiotemporal clutter filtering of ultrafast ultrasound data highly increases Doppler and fUltrasound sensitivity," IEEE Trans. Med. Imag., vol. 34, no. 11, pp. 2271-2285, 2015.

[56] E. Mace, G. Montaldo, I. Cohen, M. Baulac, M. Fink, and M. Tanter, "Functional ultrasound imaging of the brain," Nature methods, vol. 8, no. 8, pp. 662-664, 2011.

[57] O. Couture, B. Besson, G. Montaldo, M. Fink, and M. Tanter, "Microbubble ultrasound super-localization imaging (MUSLI)," in Proc. IEEE Ultrason. Symp., pp. 1285-1287, 2011.

[58] M. Siepmann, G. Schmitz, J. Bzyl, M. Palmowski, and F. Kiessling, "Imaging tumor vascularity by tracing single microbubbles," Proc. IEEE Ultrason. Symp., pp. 6293297, 1906-1908, 2011.

[59] O. M. Viessmann, R. J. Eckersley, K. Christensen-Jeffries, M. X Tang, and C. Dunsby, "Acoustic super-resolution with ultrasound and microbubbles," Phys. Med. Biol., vol. 58, pp. 6447-6458, 2013.

[60] M. A. O'Reilly and K. Hynynen, "A super-resolution ultrasound method for brain vascular mapping," Med. Phys., vol. 40, no. 11, pp. 110701-7, 2013.

[61] K. Christensen-Jeffries, R. J. Browning, M. Tang, C. Dunsby, and R. J. Eckersley, "In vivo acoustic super-resolution and super-resolved velocity mapping using microbubbles," IEEE Trans. Med. Imag., vol. 34, pp. 433-440, February 2015.

[62] C. Errico, J. Pierre, S. Pezet, Y. Desailly, Z. Lenkei, O. Couture, and M. Tanter, "Ultrafast ultrasound localization microscopy for deep superresolution vascular imaging," Nature, vol. 527, pp. 499-502, November 2015.

[63] K. Christensen-Jeffries, O. Couture, P. A. Dayton, Y. C. Eldar, K. Hynynen, F. Kiessling, M. O'Reilly, G. F. Pinton, G. Schmitz, M. X. Tang, M. Tanter, and R. J. van Sloun, "Super-resolution ultrasound imaging," Ultrasound Med. Biol., vol. 46, no. 4, pp. 865-891, 2020.

[64] S. Harput, K. Christensen-Jeffries, A. Ramalli, J. Brown, J. Zhu, G. Zhang, C. H. Leow, M. Toulemonde, E. Boni, P. Tortoli, R. J. Eckers- ley, C. Dunsby, and M. Tang, "3-D super-resolution ultrasound (SR-US) imaging with a 2-D sparse array," arXiv preprint, p. 1902.01608v1, 2019.

[65] J. A. Jensen, M. L. Ommen, S. H. Øygard, M. Schou, T. Sams, M. B. Stuart, C. Beers, E. V. Thomsen, N. B. Larsen, and B. G. Tomov, "Threedimensional super resolution imaging using a row-column array," IEEE Trans. Ultrason., Ferroelec., Freq. Contr., vol. 67, no. 3, pp. 538-546, 2020.

[66] E. Mace, G. Montaldo, B. Osmanski, I. Cohen, M. Fink, and M. Tanter, "Functional ultrasound imaging of the brain: Theory and basic principles," IEEE Trans. Ultrason., Ferroelec., Freq. Contr., vol. 60, no. 3, pp. 492-506, 2013.

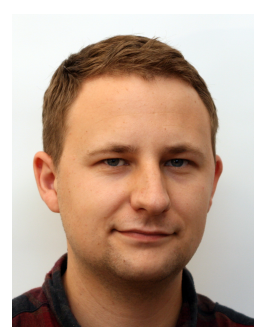

Mikkel Schou was born in 1990. He received the M.Sc. degree in biomedical engineering from the Technical University of Denmark, Kongens Lyngby, Denmark, and the University of Copenhagen, Copenhagen, Denmark, in 2017. He is currently pursuing the Ph.D. degree in biomedical engineering with the Center for Fast Ultrasound Imaging, Technical University of Denmark. The topic of his $\mathrm{Ph} . \mathrm{D}$. research is 3-D ultrasound Perfusion and Flow imaging using Row-Column Arrays.

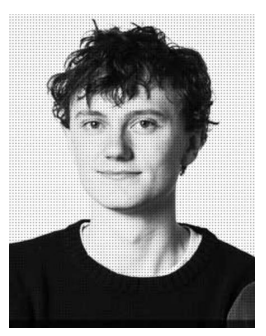

Lasse Thurmann Jørgensen received the M.S.c. Degree in biomedical engineering from the Technical University of Denmark (DTU), Lyngby, Denmark, in 2019. He is currently a Ph.D. candidate at DTU Health Tech in the Center for Fast Ultrasound imaging, where his topic of research includes motion estimation with 3-D ultrasound systems.

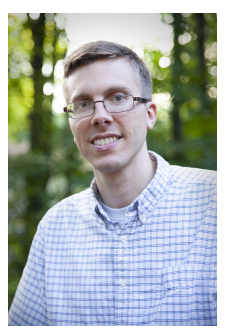

Christopher Beers received the M.S. degree in acoustics from Pennsylvania State University, State College, PA, USA, in 2007, where his thesis research explored end-element anomalies in medical ultrasound transducer arrays. He has been with BK Medical, State College, PA, USA, since 2007 (formerly Sound Technology), where he develops transducer technology and designs commercial medical ultrasound probes.

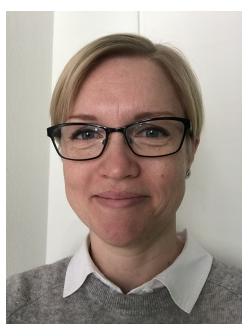

Marie Sand Traberg received her M.Sc. degree in biomedical engineering from the Technical University of Denmark and University of Copenhagen, Denmark, in July 2008. In January 2012, she earned her $\mathrm{PhD}$ degree from the Technical University of Denmark. The $\mathrm{PhD}$ thesis work was focused on predicting the biomechanical behavior of abdominal aortic aneurysms using finite element based CFD and FSI models. She holds an associate professorship in the biomedical engineering section at DTU Health Tech. Her reseach interests are development of CFD and FSI models of the cardiovascular system based on medical imaging data with specific focus on the carotid artery and the heart. 


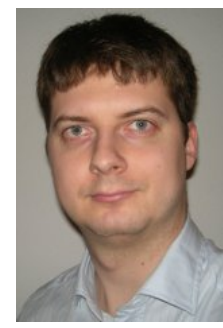

Matthias Bo Stuart eceived the M.Sc. and Ph.D. degrees in Computer Engineering from the Technical University of Denmark, Lyngby, Denmark in 2006 and 2010 respectively. He is currently an Associate Professor with the Biomedical Engineering Section, Department of Health Technology at the Technical University of Denmark. His research interests include synthetic aperture methods for both anatomical and flow imaging in both 2-D and 3-D, ultrasound systems, and real-time implementations of ultrasound processing algorithms.

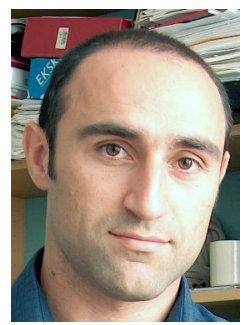

Borislav Gueorguiev Tomov received a M. Sc. degree in Electronics Engineering from the Technical University of Sofia, Bulgaria, in 1996, and a Ph.D. degree in Medical Electronics from the Technical University of Denmark in 2003. He is currently Senior Researcher at the Center for Fast Ultrasound imaging, Department of Health Technology, Technical University of Denmark. His research interests include medical ultrasound signal processing, and ultrasound scanner architectures and implementations.

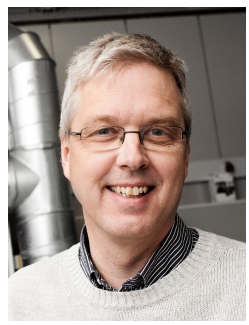

Jørgen Arendt Jensen (M'93-SM'02-F'12) received the MSc degree in 1985, the Ph.D. degree in 1989, and the Dr.Techn. degree in 1996 all from the Technical University of Denmark. Since 1993, he has been a Full Professor of Biomedical Signal Processing with the Department of Health Technology, Technical University of Denmark. He has been the founder and head of the Center for Fast Ultrasound Imaging since its inauguration in 1998. CFU has contributed with innovations in transverse oscillation vector flow imaging, synthetic aperture flow imaging in 2-D and 3-D, ultrasound simulation, research scanners, and row-column probes and beamforming. He has published more than 500 journal and conference papers on signal processing and medical ultrasound and the book Estimation of Blood Velocities Using Ultrasound (Cambridge Univ. Press), 1996. He is also the developer and maintainer of the Field II simulation program. He has been a visiting scientist at Duke University, Stanford University, and the University of Illinois at Urbana-Champaign. He was founder and head the Biomedical Engineering group from 2007 to 2010 In 2003, he was one of the founders of the biomedical engineering program in Medicine and Technology, which is a joint degree program between the Technical University of Denmark and the Faculty of Health and Medical Sciences at the University of Copenhagen. The degree is one of the most sought-after engineering degrees in Denmark. He was chairman of the study board from 2003 to 2010 and Adjunct Professor with the University of Copenhagen from 2005 to 2010 . He has given a number of short courses on simulation, synthetic aperture imaging, and flow estimation at international scientific conferences and teaches biomedical signal processing and medical imaging at the Technical University of Denmark. His research is centered around simulation of ultrasound imaging, synthetic aperture imaging, vector blood flow estimation, 3-D and super resolution imaging, row-column probes, and construction of ultrasound research systems. He has educated $43 \mathrm{PhD}$ students and currently advises $16 \mathrm{PhD}$ students. Dr. Jensen has given more than 60 invited talks at international meetings and received several awards for his research, most recently the Grand Solutions Prize from the Danish Minister of Science, the order of the Dannebrog by her Majesty the Queen of Denmark, and the Rayleigh award from the UFFC Society in the field of Ultrasonics in 2019. 